This item was submitted to Loughborough's Research Repository by the author.

Items in Figshare are protected by copyright, with all rights reserved, unless otherwise indicated.

\title{
Constrained anti-disturbance control for a quadrotor based on differential flatness
}

PLEASE CITE THE PUBLISHED VERSION

http://dx.doi.org/10.1080/00207721.2016.1244307

PUBLISHER

(c) Taylor \& Francis

VERSION

AM (Accepted Manuscript)

\section{PUBLISHER STATEMENT}

This work is made available according to the conditions of the Creative Commons Attribution-NonCommercialNoDerivatives 4.0 International (CC BY-NC-ND 4.0) licence. Full details of this licence are available at: https://creativecommons.org/licenses/by-nc-nd/4.0/

\section{LICENCE}

CC BY-NC-ND 4.0

\section{REPOSITORY RECORD}

Lu, Hao, Cunjia Liu, Lei Guo, and Wen-Hua Chen. 2016. "Constrained Anti-disturbance Control for a Quadrotor Based on Differential Flatness”. figshare. https://hdl.handle.net/2134/22910. 
To appear in the International Journal of Systems Science

Vol. 00, No. 00, Month 20XX, 1-23

\title{
GUIDE
}

\section{Constrained Anti-Disturbance Control for a Quadrotor Based on Differential Flatness}

\author{
Hao Lu ${ }^{\mathrm{a}}$, Cunjia Liu ${ }^{\mathrm{b} *}, \mathrm{Lei} \mathrm{Guo}^{\mathrm{c}}$, and Wen-Hua Chen ${ }^{\mathrm{b}, \mathrm{c}}$ \\ ${ }^{a}$ Beijing Electro-Mechanical Engineering Institute, Beijing, China; ${ }^{b}$ Department of Aeronautical and \\ Automotive Engineering, Loughborough University, Loughborough, UK; ${ }^{c}$ School of Automation Science and \\ Electrical Engineering, Beihang University, Beijing, China;
}

(v5.0 released February 2015)

\begin{abstract}
The classical control design based on linearised model is widely used in practice even to those inherently nonlinear systems. Although linear design techniques are relatively mature and enjoy the simple structure in implementations, they can be prone to misbehaviour and failure when the system state is far away from the operating point. To avoid the drawbacks and exploit the advantages of linear design methods while tackling the system nonlinearity, a hybrid control structure is developed in this paper. First, the model predictive control is used to impose states and inputs constraints on the linearised model, which makes the linearisation satisfy the small-perturbation requirement and reduces the bound of linearisation error. On the other hand, a combination of disturbance observer based control and $H_{\infty}$ control, called composite hierarchical anti-disturbance control, is constructed for the linear model to provide robustness against multiple disturbances. The constrained reference states and inputs generated by the outer-loop model predictive controller are asymptotically tracked by the inner-loop composite anti-disturbance controller. To demonstrate the performance of the proposed framework, a case study on quadrotor is conducted.
\end{abstract}

Keywords: model predictive control; online optimisation; disturbance observer based control; composite anti-disturbance control; differential flatness

\section{Introduction}

Although the nonlinear control theory has been comprehensively studied in literature over the last few decades, the classical linear control theory is still widely used in practical engineering because of the simple structure and easy implementation. The linearisation of system model at the operating point based on the small perturbation theory is the fundamental requirement to design a linear controller. In order to make the linear control algorithm available and efficient, the system states and inputs must be governed in a valid neighbourhood of the operating point such that the linearised model can maintain a good fidelity. In addition, some of the states and inputs have their own physical limits, which should also be taken into account. However, how to restrict the system states and inputs in the classical linear control approaches still remains an open problem.

Model predictive control (MPC) is popular for its ability to deal with hard constraints on inputs and states. In general, the MPC problem is formulated as solving a finite horizon open-loop optimal control problem online at each sampling instant. The obtained optimal control sequence is based on the current states and system model. Only the first portion of this sequence is applied to the system until next sampling instant (Chen, Ballance, \& Gawthrop, 2003; Mayne, Rawlings, Rao, \& Scokaert, 2000). Solving a nonlinear optimisation problem with constraints is computationally intensive, which is the main obstacle that blocks the real-time application of MPC to

*Corresponding author. Email: c.liu5@lboro.ac.uk 
nonlinear systems. To reduce the computational burden of online optimisation, the differential flatness property has been exploited in many trajectory planning algorithms which are actually MPC problems (Berry, Howitt, Gu, \& Postlethwaite, 2011; Cowling, Yakimenko, Whidborne, \& Cooke, 2010; Faulwasser, Hagenmeyer, \& Findeisen, 2014; Flores \& Milam, 2006; Mahadevan, Agrawal, \& Doyle, 2001; Prodan et al., 2013; Suryawan, De Don, \& Seron, 2012). Loosely speaking, the differential flatness means that the flat system can be completely characterised by flat outputs and their higher order derivatives (Fliess, Lvine, Martin, \& Rouchon, 1995). Thus, an infinite dimensional dynamic optimal control problem can be cast into a finite dimensional static one on the condition that the flat outputs are parametrised in terms of polynomials. As a result, for example, the optimisation problem reduces to a tractable nonlinear programming (NLP) problem (Cowling et al., 2010) or even a simpler quadratic programming (QP) problem (Suryawan et al., 2012). Although the reduction in calculation time of the optimisation can bring a great increase to the updated rate of MPC, this kind of nonlinear constrained optimal control may still result in a degraded performance due to the disturbances and uncertainties existing in practical application process, especially for the sophisticated systems with relatively fast dynamics like aircraft. Therefore, it is essential to incorporate with another robust control approach.

Disturbances originated from various sources, such as modelling errors, parameter uncertainties, and external environment, widely exist in practical systems, which may cause significant adverse effects on the performance and even the stability of the control system. To achieve high-accuracy control, disturbance attenuation and rejection are actually the key objectives in control system design (Chen, Yang, Guo, \& Li, 2015). $H_{\infty}$ control, the common representative of disturbance attenuation algorithms, can suppress the influences from disturbances to controlled output to a desired level. Robust MPC methods are also discussed in He, Ji, \& Yu (2013) and He, Huang, \& Chen (2014) to attenuate the unknown bounded disturbances. Disturbance rejection strategies, such as disturbance observer based control (DOBC), estimate the influence of unknown disturbances from the measurable variables and then design a feedforward control action for direct compensation (Guo $\&$ Chen, 2005). Both disturbance attenuation and rejection can achieve good robustness against disturbances and uncertainties, however, they also have shortcomings. Disturbance attenuation is based on the worst case which may be too conservative to provide highly accurate control performance whereas the disturbance rejection approach is limited to the disturbances with bounded variation or the harmonic disturbances. Thus, to improve accuracy, both the disturbance rejection and attenuation performance should be achieved simultaneously. In recent years, a robust control method, called composite hierarchical anti-disturbance control (CHADC), is proposed to cope with the multiple disturbances in complex systems (Guo \& Cao, 2013). The CHADC approach, in general, employs two layers. The inner layer rejects the disturbances with partially known information in the feedforward channel based on the disturbances estimation; meanwhile, the outer layer attenuates the remaining part without adequate knowledge in the feedback path by exploiting the disturbance attenuation strategy. The state-of-the-art CHADC approach has been well developed on the linearised system with nonlinearity, of which the nonlinearity is the error of linearisation at a certain operating point. The feedback $H_{\infty}$ control, sliding model control, and adaptive control have been integrated respectively with the feedforward DOBC to form this type of CHADC strategy (Guo \& Wen, 2011; Peng, Fang, \& Xu, 2015; Wei \& Guo, 2010; Wei, Zhang, \& Guo, 2009; Yao \& Guo, 2013; Yang, Li, \& Yu, 2013).

In this paper, we propose a constrained anti-disturbance control scheme which combines MPC and CHADC together. The MPC design deals with the nominal nonlinear dynamics without disturbances. It works in the outer-loop to provide the reference states and the corresponding inputs that satisfy system constraints. In the inner-loop, the CHADC, constructed based on the linearised model with disturbances, forces the system to follow the constrained reference states and correct the reference inputs. As a constrained anti-disturbance controller, it not only satisfies the hard constraints but also provides robustness against multiple disturbances within its capability. To demonstrate the effectiveness of the proposed control strategy, a quadrotor is adopted as a case 
study throughout the paper. The main contributions of this paper include three aspects. Firstly, compared to the conventional linear control methods, the states and inputs constraints are taken into consideration. Restricting the reference states and inputs generated by the outer-loop MPC in a certain feasible neighbourhood of the operating point provides a feasible way to satisfy the small perturbation condition. Secondly, the bounds of the nonlinearity can be explicitly given, which reduces the design conservatism of CHADC. Thirdly, the online MPC exploits the original nonlinear dynamics, which implies that the proposed control scheme is actually a robust nonlinear control algorithm that can function on the entire trajectory continuously. Although demonstrated through a case study on the trajectory tracking control of a quadrotor, the proposed constrained anti-disturbance control scheme is readily applied to other differential flat systems as catalogued in Murray, Rathinam, \& Sluis (1995), such as manipulators, land vehicles, maglev systems, cranes, etc., after necessary modifications.

The remainder of this paper is organised as follows. Section 2 introduces the mathematical model of the quadrotor. In Section 3, the specific problems that will be discussed in this paper are formulated. The design of the outer-loop model predictive controller is detailed in Section 4 . Section 5 is devoted to the design of composite hierarchical anti-disturbance controller. Section 6 provides the simulation results to demonstrate the effectiveness of the proposed approach, followed by conclusions in Section 7.

\section{Quadrotor modelling}

The quadrotor is a typical vertical take-off and landing (VTOL) unmanned aerial vehicle (UAV). Its standard configuration is briefly shown in Fig. 1. There are two coordinate systems employed

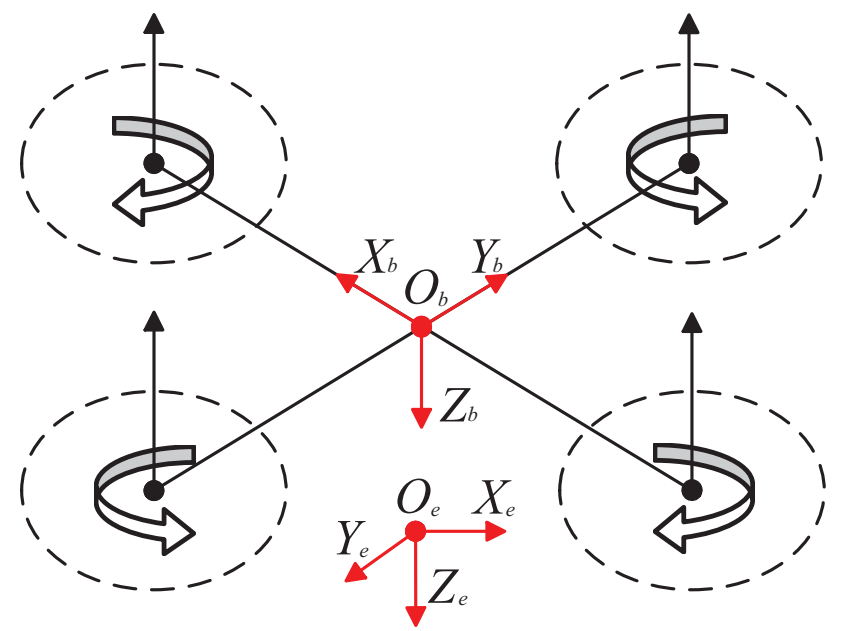

Figure 1. Quadrotor configuration

where $\boldsymbol{S}_{\mathrm{b}}=\left\{\begin{array}{lllll}O_{\mathrm{b}} & \overrightarrow{\boldsymbol{X}_{\mathrm{b}}} & \overrightarrow{\boldsymbol{Y}_{\mathrm{b}}} & \overrightarrow{\boldsymbol{Z}_{\mathrm{b}}}\end{array}\right\}$ denotes the body-fixed coordinate frame with origin at the

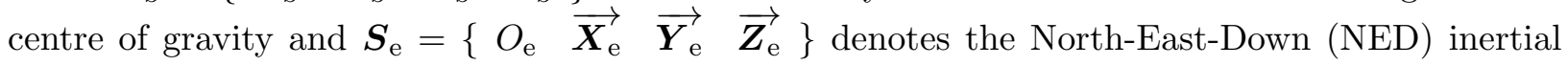
coordinate frame. Based on the Newton-Euler equations, the rigid-body translational dynamics and the rotational dynamics driven by external force $\boldsymbol{F} \in \mathbb{R}^{3}$ and torque $\boldsymbol{M} \in \mathbb{R}^{3}$ can be derived as follows (Kendoul, Lara, Fantoni-Coichot, \& Lozano, 2007)

$$
\left\{\begin{array}{l}
m \ddot{\boldsymbol{\zeta}}=m g \boldsymbol{Z}_{\mathrm{e}}+\mathbf{R} \boldsymbol{F} \\
J \dot{\boldsymbol{\Omega}}=-\boldsymbol{\Omega} \times J \boldsymbol{\Omega}+\boldsymbol{M}
\end{array}\right.
$$


where $\boldsymbol{\zeta}=\left[\begin{array}{lll}x & y & z\end{array}\right]^{\mathrm{T}}$ denote the inertial positions and $\boldsymbol{\Omega}=\left[\begin{array}{lll}p & q & r\end{array}\right]^{\mathrm{T}}$ represent the angular rates. $m, g$ and $J \in \mathbb{R}^{3}$ are the quadrotor's mass, acceleration of gravity, and diagonal inertia matrix, respectively.

$\mathbf{R}$ is the rotation matrix from body-fixed frame to inertial frame. Because the first rotation about the axis of thrust can simplify the equations of motion, rotation matrix $\mathbf{R}$ in the order of $z-x-y$ is used in this paper (Cowling et al., 2010). $\mathbf{R}_{z x y}$ is given by

$$
\mathbf{R}_{z x y}=\left[\begin{array}{ccc}
\mathrm{c} \theta \mathrm{c} \psi+\mathrm{s} \theta \mathrm{s} \phi \mathrm{s} \psi & -\mathrm{c} \theta \mathrm{s} \psi+\mathrm{s} \phi \mathrm{s} \theta \mathrm{c} \psi & \mathrm{s} \theta \mathrm{c} \phi \\
\mathrm{c} \phi \mathrm{s} \psi & \mathrm{c} \phi \mathrm{c} \psi & -\mathrm{s} \phi \\
-\mathrm{s} \theta \mathrm{c} \psi+\mathrm{s} \phi \mathrm{c} \theta \mathrm{s} \psi & \mathrm{s} \theta \mathrm{s} \psi+\mathrm{s} \phi \mathrm{c} \theta \mathrm{c} \psi & \mathrm{c} \theta \mathrm{c} \phi
\end{array}\right]
$$

where the notations $\mathrm{s}$ and $\mathrm{c}$ are abbreviations for $\sin (\cdot)$ and $\cos (\cdot)$, respectively.

The external force $\boldsymbol{F}$ and torque $\boldsymbol{M}$ are composed of two parts: one is the control force $\boldsymbol{F}_{0}$ and torque $\boldsymbol{M}_{0}$ generated by the four rotors; and another is the lumped disturbance force $\boldsymbol{F}_{\mathrm{d}}$ and torque $\boldsymbol{M}_{\mathrm{d}}$ originated from other force and torque contributions such as wind turbulences and parameter uncertainties. These relations are expressed as

$$
\begin{gathered}
\boldsymbol{F}=\underbrace{\left[\begin{array}{lll}
0 & 0 & -u
\end{array}\right]^{\mathrm{T}}}_{\boldsymbol{F}_{0}}+\underbrace{\left[\begin{array}{lll}
d_{x} & d_{y} & d_{z}
\end{array}\right]^{\mathrm{T}}}_{\boldsymbol{F}_{\mathrm{d}}}, \\
\boldsymbol{M}=\underbrace{\left[\begin{array}{lll}
u_{p} & u_{q} & u_{r}
\end{array}\right]^{\mathrm{T}}}_{\boldsymbol{M}_{0}}+\underbrace{\left[\begin{array}{lll}
d_{p} & d_{q} & d_{r}
\end{array}\right]^{\mathrm{T}}}_{\boldsymbol{M}_{\mathrm{d}}}
\end{gathered}
$$

where $u$ is the main thrust and $u_{p}, u_{q}$, and $u_{r}$ are the control torques. They are produced by altering the angular velocities of the four rotors:

$$
\left[\begin{array}{c}
u \\
u_{p} \\
u_{q} \\
u_{r}
\end{array}\right]=\left[\begin{array}{cccc}
\rho & \rho & \rho & \rho \\
0 & -\rho l & 0 & \rho l \\
-\rho l & 0 & \rho l & 0 \\
\kappa & -\kappa & \kappa & -\kappa
\end{array}\right]\left[\begin{array}{c}
w_{1}^{2} \\
w_{2}^{2} \\
w_{3}^{2} \\
w_{4}^{2}
\end{array}\right]
$$

where $l$ is the distance from the rotor to the centre of cross frame; $\rho$ and $\kappa$ are the propeller-to-force and propeller-to-torque scaling factors, respectively. The propellers are driven by DC motors and $w_{\mathrm{i}}, \mathrm{i}=1,2,3,4$ are the motor velocities.

To facilitate the flight control design, the rotational dynamics are further explored. The rotational kinematics relationship between the angular rates $(p, q, r)$ and the generalised velocities $(\dot{\phi}, \dot{\theta}, \dot{\psi})$ can be derived as

$$
\underbrace{\left[\begin{array}{c}
p \\
q \\
r
\end{array}\right]}_{\boldsymbol{\Omega}}=\underbrace{\left[\begin{array}{ccc}
\cos \psi & \cos \phi \sin \psi & 0 \\
-\sin \psi & \cos \phi \cos \psi & 0 \\
0 & -\sin \phi & 1
\end{array}\right]}_{\Psi(\boldsymbol{\eta})} \underbrace{\left[\begin{array}{c}
\dot{\phi} \\
\dot{\theta} \\
\dot{\psi}
\end{array}\right]}_{\dot{\boldsymbol{\eta}}},
$$

or compactly,

$$
\boldsymbol{\Omega}=\Psi(\boldsymbol{\eta}) \dot{\boldsymbol{\eta}}
$$

This relationship also implies $\dot{\boldsymbol{\eta}}=\Phi(\boldsymbol{\eta}) \boldsymbol{\Omega}$ where $\Phi(\boldsymbol{\eta})=\Psi^{-1}(\boldsymbol{\eta})$. Differentiating $\dot{\boldsymbol{\eta}}$ and invoking the rotational dynamics (1b), the quadrotor's rotational dynamics can be rewritten in terms of 
attitude angle vector $\boldsymbol{\eta}$ as

$$
\ddot{\boldsymbol{\eta}}=\mathbb{J}^{-1}(\boldsymbol{\eta})(\boldsymbol{M}-C(\dot{\boldsymbol{\eta}}, \boldsymbol{\eta}) \dot{\boldsymbol{\eta}})
$$

where $\mathbb{J}(\boldsymbol{\eta})=J \Psi(\boldsymbol{\eta})$ is defined as a pseudo-inertia matrix and $C(\dot{\boldsymbol{\eta}}, \boldsymbol{\eta})=\dot{\mathbb{J}}(\boldsymbol{\eta})+\Psi(\boldsymbol{\eta}) \times \mathbb{J} \dot{\boldsymbol{\eta}}$ is the Coriolis term (Kendoul et al., 2007). Considering the two ingredients of the external torque $M$ in Eq. (2b) separately, $\mathbb{J}^{-1}(\boldsymbol{\eta})\left(\boldsymbol{M}_{0}-C(\dot{\boldsymbol{\eta}}, \boldsymbol{\eta}) \dot{\boldsymbol{\eta}}\right)$ is defined as a new pseudo-control toques $\overline{\boldsymbol{M}}_{0}=$ $\left[\begin{array}{lll}u_{\phi} & u_{\theta} & u_{\psi}\end{array}\right]^{\mathrm{T}}$ and the disturbance torque $\boldsymbol{M}_{\mathrm{d}}$ also can be converted into $\overline{\boldsymbol{M}}_{\mathrm{d}}=\left[\begin{array}{lll}d_{\phi} & d_{\theta} & d_{\psi}\end{array}\right]^{\mathrm{T}}$ such that Eq. (4) is written as follows

$$
\ddot{\eta}=\bar{M}_{0}+\bar{M}_{\mathrm{d}}
$$

Combining the translational dynamics (1a) and modified rotational dynamics (5), the quadrotor model can be expressed by

$$
\left\{\begin{array}{l}
\ddot{x}=-\frac{u}{m} \cos \phi \sin \theta+d_{x} \\
\ddot{y}=\frac{u}{m} \sin \phi+d_{y} \\
\ddot{z}=-\frac{u}{m} \cos \phi \cos \theta+g+d_{z} \\
\ddot{\phi}=u_{\phi}+d_{\phi} \\
\ddot{\theta}=u_{\theta}+d_{\theta} \\
\ddot{\psi}=u_{\psi}+d_{\psi}
\end{array}\right.
$$

where $\boldsymbol{x}=\left[\begin{array}{llllllllllll}x & y & z & \dot{x} & \dot{y} & \dot{z} & \phi & \theta & \psi & \dot{\phi} & \dot{\theta} & \dot{\psi}\end{array}\right]^{\mathrm{T}}$ is the 12-dimensional state vector. $\boldsymbol{u}=$ $\left[\begin{array}{llll}u & u_{\phi} & u_{\theta} & u_{\psi}\end{array}\right]^{\mathrm{T}}, \boldsymbol{y}=\left[\begin{array}{llll}x & y & z & \psi\end{array}\right]^{\mathrm{T}}$, and $\boldsymbol{d}=\left[\begin{array}{llllll}d_{x} & d_{y} & d_{z} & d_{\phi} & d_{\theta} & d_{\psi}\end{array}\right]^{\mathrm{T}}$ are defined as the vectors of input, output, and lumped disturbance, respectively.

Remark 1: The pseudo-control torques $\overline{\boldsymbol{M}}_{0}=\left[\begin{array}{lll}u_{\phi} & u_{\theta} & u_{\psi}\end{array}\right]^{\mathrm{T}}$ are introduced to simplify the control design procedure, but they are not the actual control torques. The actual control torques $\boldsymbol{M}_{0}=\left[\begin{array}{lll}u_{p} & u_{q} & u_{r}\end{array}\right]^{\mathrm{T}}$ can be reversely derived from $\overline{\boldsymbol{M}}_{0}=\mathbb{J}^{-1}(\boldsymbol{\eta})\left(\boldsymbol{M}_{0}-C(\dot{\boldsymbol{\eta}}, \boldsymbol{\eta}) \dot{\boldsymbol{\eta}}\right)$.

\section{Problem formulation}

The quadrotor model (6) can be put into the following general nonlinear form with lumped disturbance $\boldsymbol{d}$ :

$$
\dot{\boldsymbol{x}}=f(\boldsymbol{x}, \boldsymbol{u})+\mathbf{B}_{\mathrm{d}} \boldsymbol{d} .
$$

By linearising the quadrotor model at the operating point $\left(\boldsymbol{x}_{0}, \boldsymbol{u}_{0}\right)$ according to the small perturbation theory, a linear time-invariant error dynamics model with linearisation error $\Delta f(\tilde{\boldsymbol{x}}, \tilde{\boldsymbol{u}})$ is derived as follows

$$
\dot{\tilde{\boldsymbol{x}}}=\mathbf{A} \tilde{\boldsymbol{x}}+\mathbf{B} \tilde{\boldsymbol{u}}+\mathbf{B}_{\mathrm{d}} \boldsymbol{d}+\Delta f(\tilde{\boldsymbol{x}}, \tilde{\boldsymbol{u}})
$$

where $\tilde{\boldsymbol{x}}=\boldsymbol{x}-\boldsymbol{x}_{0}$ and $\tilde{\boldsymbol{u}}=\boldsymbol{u}-\boldsymbol{u}_{0}$ are defined as the errors between the actual state and input and the operating point. $\mathbf{A}=\left.\frac{\partial f}{\partial \boldsymbol{x}}\right|_{\boldsymbol{x}_{0}, \boldsymbol{u}_{0}}$ and $\mathbf{B}=\left.\frac{\partial f}{\partial \boldsymbol{u}}\right|_{\boldsymbol{x}_{0}, \boldsymbol{u}_{0}}$ are the Jacobian matrices and the coefficient 
matrix of disturbance $\boldsymbol{d}$ is

$$
\mathbf{B}_{\mathrm{d}}=\left[\begin{array}{ll}
\boldsymbol{0}_{3 \times 3} & \mathbf{0}_{3 \times 3} \\
\boldsymbol{I}_{3 \times 3} & \mathbf{0}_{3 \times 3} \\
\mathbf{0}_{3 \times 3} & \mathbf{0}_{3 \times 3} \\
\mathbf{0}_{3 \times 3} & \boldsymbol{I}_{3 \times 3}
\end{array}\right] .
$$

This linearised model presents the dynamic response of the perturbed system state vector from the specified operating condition. Considering the hovering condition $\left(\phi_{0}=0, \theta_{0}=0, u_{0}=m g\right)$ as the operating condition yields

$$
\mathbf{A}=\left[\begin{array}{cccccc}
\mathbf{0}_{3 \times 3} & \mathbf{I}_{3 \times 3} & \mathbf{0}_{3 \times 1} & \mathbf{0}_{3 \times 1} & \mathbf{0}_{3 \times 1} & \mathbf{0}_{3 \times 3} \\
\mathbf{0}_{1 \times 3} & \mathbf{0}_{1 \times 3} & 0 & -g & 0 & \mathbf{0}_{1 \times 3} \\
\mathbf{0}_{1 \times 3} & \mathbf{0}_{1 \times 3} & g & 0 & 0 & \mathbf{0}_{1 \times 3} \\
\mathbf{0}_{1 \times 3} & \mathbf{0}_{1 \times 3} & 0 & 0 & 0 & \mathbf{0}_{1 \times 3} \\
\mathbf{0}_{3 \times 3} & \mathbf{0}_{3 \times 3} & \mathbf{0}_{3 \times 1} & \mathbf{0}_{3 \times 1} & \mathbf{0}_{3 \times 1} & \mathbf{I}_{3 \times 3} \\
\mathbf{0}_{3 \times 3} & \mathbf{0}_{3 \times 3} & \mathbf{0}_{3 \times 1} & \mathbf{0}_{3 \times 1} & \mathbf{0}_{3 \times 1} & \mathbf{0}_{3 \times 3}
\end{array}\right] \text { and } \mathbf{B}=\left[\begin{array}{cc}
\mathbf{0}_{3 \times 1} & \mathbf{0}_{3 \times 3} \\
0 & \mathbf{0}_{1 \times 3} \\
0 & \mathbf{0}_{1 \times 3} \\
-\frac{1}{m} & \mathbf{0}_{1 \times 3} \\
\mathbf{0}_{3 \times 1} & \mathbf{0}_{3 \times 3} \\
\mathbf{0}_{3 \times 1} & \mathbf{I}_{3 \times 3}
\end{array}\right] .
$$

To design a linear controller for system (8), it is essential to make the following assumption.

Assumption 1: For any $\boldsymbol{x} \in \boldsymbol{D}_{1}$ and $\boldsymbol{u} \in \boldsymbol{D}_{2}, \boldsymbol{D}_{1} \in \boldsymbol{R}^{n}$ and $\boldsymbol{D}_{2} \in \boldsymbol{R}^{m}$ are the definitional domains, the nonlinear error function $\Delta f(\tilde{\boldsymbol{x}}, \tilde{\boldsymbol{u}})$ satisfies

$$
\|\Delta f(\tilde{\boldsymbol{x}}, \tilde{\boldsymbol{u}})\| \leq\|\mathbf{U} \tilde{\boldsymbol{x}}\|
$$

where $\mathbf{U}$ is a constant weighting matrix.

This assumption can be described as the bound condition for the linearisation error $\Delta f$. If we traverse all the actual values of $\boldsymbol{x}$ and $\boldsymbol{u}$ in the domains to search for the appropriate $\mathbf{U}$, the obtained result will be conservative and impractical. For the quadrotor, the roll and pitch attitude angles always need to be restricted in a safe operating region, usually $\left[-45^{\circ}, 45^{\circ}\right]$ set in the commercialoff-the-shelf autopilot, which can also make the linearised model maintain a reasonable fidelity. In addition, the thrust $u>0$ is required to avoid free fall and it is also upper bounded by $u_{\max }$. Thus, the following constraints are enforced on the quadrotor dynamics:

$$
-45^{\circ} \leq \phi \leq 45^{\circ},-45^{\circ} \leq \theta \leq 45^{\circ}, 0<u \leq u_{\max },
$$

from which the corresponding weighting matrix $\mathbf{U}$ can be calculated.

Stabilising system (8) at the operating point is a regulation problem. In order to achieve tracking, the actual control signal is constructed as (Cowling et al., 2010; Raptis, Valavanis, \& Vachtsevanos, 2012):

$$
\boldsymbol{u}=\boldsymbol{u}_{\mathrm{ref}}+\tilde{\boldsymbol{u}}_{\mathrm{fb}}
$$

where $\tilde{\boldsymbol{u}}_{\mathrm{fb}}$ is the feedback control law that renders the following error dynamics

$$
\dot{\tilde{\boldsymbol{x}}}_{\mathrm{e}}=\mathbf{A} \tilde{\boldsymbol{x}}_{\mathrm{e}}+\mathbf{B} \tilde{\boldsymbol{u}}_{\mathrm{fb}}+\mathbf{B}_{\mathrm{d}} \boldsymbol{d}+\Delta f\left(\tilde{\boldsymbol{x}}_{\mathrm{e}}, \tilde{\boldsymbol{u}}_{\mathrm{fb}}\right)
$$

asymptotically stable. $\tilde{\boldsymbol{x}}_{e}=\boldsymbol{x}-\boldsymbol{x}_{\mathrm{ref}}$ is the error between the actual state and its desired value. The coefficient matrices $\mathbf{A}$ and $\mathbf{B}$ derived from hovering condition are retained in Eq. (12). Thus, the parameter errors are incorporated into the nonlinear error function $\Delta f\left(\tilde{\boldsymbol{x}}_{\mathrm{e}}, \tilde{\boldsymbol{u}}_{\mathrm{fb}}\right)$. The discussions on how to design the reference pair $\left(\boldsymbol{x}_{\text {ref }}, \boldsymbol{u}_{\text {ref }}\right)$ that subjects to the constraints when tracking the 
position command and the feedback corrective term $\tilde{\boldsymbol{u}}_{\mathrm{fb}}$ that is robust against disturbances will be detailed in the following sections.

\section{Constrained model predictive control design}

\subsection{Model predictive control formulation}

In this section, a model predictive controller is designed to deal with the nominal nonlinear system without lumped disturbances. It is implemented in the outer-loop to generate the constrained reference trajectory $\boldsymbol{x}_{\text {ref }}$ and control input $\boldsymbol{u}_{\text {ref }}$.

The objective of autonomous flight control is steering the quadrotor to track a predefined reference trajectory. Defining the reference trajectory as $\boldsymbol{y}_{\mathrm{r}}=\left[\begin{array}{llll}x_{\mathrm{r}} & y_{\mathrm{r}} & z_{\mathrm{r}} & \psi_{\mathrm{r}}\end{array}\right]^{\mathrm{T}}$, the cost function designed for minimising the tracking errors is given by

$$
J(t)=\left\|\boldsymbol{y}(t+T)-\boldsymbol{y}_{\mathrm{r}}(t+T)\right\|_{\mathbf{Q}}^{2}+\int_{t}^{t+T}\left(\left\|\boldsymbol{y}(\tau)-\boldsymbol{y}_{\mathrm{r}}(\tau)\right\|_{\mathbf{R}}^{2}+\|\boldsymbol{\eta}(\tau)\|_{\mathbf{S}}^{2}\right) d \tau
$$

where the notation $\|\boldsymbol{x}\|_{\mathbf{Q}}^{2}$ represents $\boldsymbol{x}^{T} \mathbf{Q} \boldsymbol{x} ; \mathbf{Q}, \mathbf{R}$, and $\mathbf{S}$ denote positive definite weighting matrices for terminal cost, stage cost, and input cost, respectively. $T$ is the prediction horizon. Since the MPC works as an outer-loop controller, the attitude angle $\boldsymbol{\eta}$ can be regarded as its control input. Taking into account the system dynamics and constraints, the optimal control problem at current sampling instant $t$ can be formulated into the following form:

$$
\min _{\boldsymbol{y}} J(t)
$$

subject to:

$$
\begin{aligned}
& \dot{\boldsymbol{x}}(\tau)=f(\boldsymbol{x}(\tau), \boldsymbol{u}(\tau)), \boldsymbol{y}(\tau)=h(\boldsymbol{x}(\tau)), \tau \in[t, t+T] \\
& \boldsymbol{x}_{0}=\boldsymbol{x}(t) \\
& \boldsymbol{x}_{\mathrm{lb}} \leq \boldsymbol{x}(\tau) \leq \boldsymbol{x}_{\mathrm{ub}}, \tau \in[t, t+T] \\
& \boldsymbol{u}_{\mathrm{lb}} \leq \boldsymbol{u}(\tau) \leq \boldsymbol{u}_{\mathrm{ub}}, \tau \in[t, t+T]
\end{aligned}
$$

where Eq. (15a) is the general nonlinear form of the quadrotor model (6) without disturbances. $\boldsymbol{x}(\tau)$ is the system state driven by the control input $\boldsymbol{u}(\tau)$ and the output $\boldsymbol{y}(\tau)$ is derived from a function of $\boldsymbol{x}(\tau)$. Eq. (15b) is the initial condition, and inequality constraints (10) on states and inputs are expressed through Eq. (15c) and Eq. (15d). The next sampling time to repeat this optimal calculation is $t+\delta$, where $\delta$ is the sampling interval. Since the optimal control problem is formulated based on the current system state and repeatedly solved in a receding horizon framework, this model predictive controller is actually a closed-loop controller.

Directly solving the optimal control problem (14) is an intractable task because one must seek for the minimum in an infinite-dimensional space. To overcome this difficulty, the differential flatness property and polynomial parametrisation are exploited to characterise the optimal control problem by using finite number of parameters.

\subsection{Differential flatness based transformation}

Differential flatness is a property of some nonlinear dynamic systems, for which all the system states and inputs can be expressed by a set of specific variables, namely the flat outputs, and their 
derivatives up to some finite orders (Fliess et al., 1995):

$$
\begin{aligned}
\boldsymbol{x}(t) & =\Upsilon\left(\boldsymbol{z}(t), \dot{\boldsymbol{z}}(t), \ddot{\boldsymbol{z}}(t), \cdots, \boldsymbol{z}^{(r)}(t)\right), \\
\boldsymbol{u}(t) & =\Gamma\left(\boldsymbol{z}(t), \dot{\boldsymbol{z}}(t), \ddot{\boldsymbol{z}}(t), \cdots, \boldsymbol{z}^{(r+1)}(t)\right)
\end{aligned}
$$

where $\boldsymbol{z}(t)$ is a vector of the flat outputs and it has the same number of elements with the input vector $\boldsymbol{u}(t)$.

As for the nominal quadrotor dynamics (6) in absence of disturbances, the system output vector $\boldsymbol{y}=\left[\begin{array}{llll}x & y & z & \psi\end{array}\right]^{\mathrm{T}}$ is chosen as the flat output $\boldsymbol{z}$. The attitude angles and control inputs can be expressed in terms of the flat outputs and their derivatives, such that

$$
\left\{\begin{array}{l}
\phi=\sin ^{-1}\left(\frac{\ddot{y}}{\sqrt{\ddot{x}^{2}+\ddot{y}^{2}+(\ddot{z}-g)^{2}}}\right) \\
\theta=\tan ^{-1}\left(\frac{\ddot{x}}{\ddot{z}-g}\right) \\
\psi=\psi
\end{array}\right.
$$

and

$$
\left\{\begin{aligned}
u & =m \sqrt{\ddot{x}^{2}+\ddot{y}^{2}+(\ddot{z}-g)^{2}} \\
u_{\phi} & =\ddot{\phi}, u_{\theta}=\ddot{\theta}, u_{\psi}=\ddot{\psi} .
\end{aligned}\right.
$$

Moreover, the first-order and second-order derivatives of $\phi$ and $\theta$ can be further derived from Eqs. (17a) and (17b) by continuous differentiation and substitution (Lu, Liu, Coombes, Guo, \& Chen, 2016). Usually, the MATLAB Symbolic Math Toolbox is used to facilitate such kind of derivative calculation. The constraint of thrust $u$ in (10) avoids singularities, namely $\ddot{z}=g$, appearing in Eqs. (17a) and (17b). Substituting Eqs. (17a), (17b), and (18a) into constraints (10) yields inequalities

$$
\left\{\begin{array}{rl}
-\frac{\sqrt{2}}{2} & \leq \frac{\ddot{y}}{\sqrt{\ddot{x}^{2}+\ddot{y}^{2}+(\ddot{z}-g)^{2}}} \leq \frac{\sqrt{2}}{2} \\
-1 & \leq \frac{\ddot{x}}{\ddot{z}-g} \leq 1 \\
0 & <m \sqrt{\ddot{x}^{2}+\ddot{y}^{2}+(\ddot{z}-g)^{2}} \leq u_{\max }
\end{array} .\right.
$$

Solving the inequalities, a feasible subset of all the possible solutions can be derived as follows

$$
\left\{\begin{array}{l}
\ddot{x}+\ddot{z} \leq g \\
\ddot{x}-\ddot{z} \geq-g \\
\ddot{y}+\ddot{z} \leq g \\
\ddot{y}-\ddot{z} \geq-g \\
-g \leq \ddot{z}<g
\end{array}\right.
$$

where we restrict the altitude acceleration $\ddot{z}$ to $[-g, g)$. It can be seen that the constraints of attitude angles and thrust are transformed to be imposed on the translational accelerations due to the differential flatness property. Moreover, we can also replace the attitude angle $\boldsymbol{\eta}$ by the translational acceleration $\ddot{\boldsymbol{\zeta}}$, expressed in terms of $\ddot{z}$, in the cost function (13) such that the cost 
function becomes

$$
J(t)=\left\|\boldsymbol{z}(t+T)-\boldsymbol{z}_{\mathrm{r}}(t+T)\right\|_{\mathbf{Q}}^{2}+\int_{t}^{t+T}\left(\left\|\boldsymbol{z}(\tau)-\boldsymbol{z}_{\mathrm{r}}(\tau)\right\|_{\mathbf{R}}^{2}+\|\ddot{\boldsymbol{z}}(\tau)\|_{\mathbf{S}}^{2}\right) d \tau
$$

By using the differential flatness property, the system behaviours are described in the flat output space. Thus, with this transformation the optimal control problem (14)-(15) can be expressed as

$$
\min _{z} J(t)
$$

subject to:

$$
\begin{aligned}
& \boldsymbol{c}_{0}(\overline{\boldsymbol{z}}(t))=\boldsymbol{x}(t) \\
& \boldsymbol{l} \boldsymbol{b}_{\tau} \leq \boldsymbol{c}_{\tau}(\overline{\boldsymbol{z}}(\tau)) \leq \boldsymbol{u} \boldsymbol{b}_{\tau}
\end{aligned}
$$

where $\overline{\boldsymbol{z}}(t)=(\boldsymbol{z}(t), \dot{\boldsymbol{z}}(t), \ddot{\boldsymbol{z}}(t))$. Since the flat outputs can characterise the system dynamics inherently, the dynamic constraint Eq. (15a) in the original OC problem has been removed. The original inequality constraints (15c) and (15d) can be replaced by a linear one (23b) in terms of $\overline{\boldsymbol{z}}(t)$ which also is the compact form of (20).

\subsection{Parametrisation using B-spline polynomials}

Furthermore, the B-spline polynomials are adopted to parametrise the flat outputs over the space of basis functions. A $\mu$-th degree $\mathrm{B}$-spline curve $C(\epsilon)$ is a piecewise polynomial function represented by (Piegl \& Tiller, 1997)

$$
C(\epsilon)=\sum_{i=1}^{n} N_{i, \mu}(\epsilon) P_{i}, \quad 0 \leq \epsilon \leq 1
$$

where $P_{i}, i=1, \cdots, n$ are the control points and $N_{i, \mu}(\epsilon)$ are the piecewise basis polynomial functions defined on a non-decreasing knot sequence

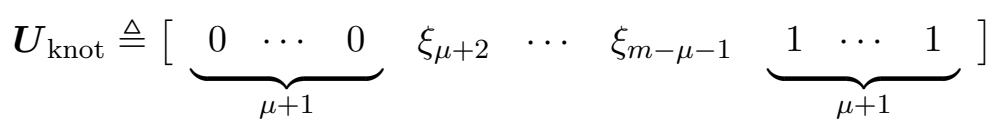

where $\xi_{k}, k=1, \cdots, m$ are called knots, and the first and last knots have multiplicity $\mu+1$. The degree of basis function $\mu$, the number of control points $n$, and the number of knots $m$ are related by

$$
m=\mu+n+1
$$

The $i$-th $\mathrm{B}$-spline basis function of $\mu$-degree is defined as

$$
\begin{aligned}
& N_{i, 0}(\epsilon)= \begin{cases}1 & \xi_{i}<\epsilon<\xi_{i+1} \\
0 & \text { otherwise }\end{cases} \\
& N_{i, \mu}(\epsilon)=\frac{\epsilon-\xi_{i}}{\xi_{i+\mu}-\xi_{i}} N_{i, \mu-1}(\epsilon)+\frac{\xi_{i+\mu+1}-\epsilon}{\xi_{i+\mu+1}-\xi_{i+1}} N_{i+1, \mu-1}(\epsilon)
\end{aligned}
$$


and the $r$-th time derivatives of the basis functions $N_{i, \mu}(\epsilon)$ is given by

$$
N_{i, \mu}^{(r)}(\epsilon)=\mu\left(\frac{N_{i, \mu-1}^{(r-1)}(\epsilon)}{\xi_{i+\mu}-\xi_{i}}-\frac{N_{i+1, \mu-1}^{(r-1)}(\epsilon)}{\xi_{i+\mu+1}-\xi_{i+1}}\right) .
$$

Remark 2: It should be noted that the derivative order $r$ can not exceed the selected B-spline curve degree $\mu$. When the denominator involving knot difference becomes zero, the quotient is defined to be zero.

Based on above definitions, the flat output vector $\boldsymbol{z}(\tau)=\left[\begin{array}{llll}x & y & z & \psi\end{array}\right]^{\mathrm{T}}$ can be parametrised in terms of the B-spline basis functions as

$$
\begin{aligned}
\boldsymbol{z}(\tau) & =\left[\begin{array}{llll}
z_{1}(\tau) & z_{2}(\tau) & z_{3}(\tau) & z_{4}(\tau)
\end{array}\right]^{\mathrm{T}} \\
& =\left[\begin{array}{llll}
\boldsymbol{N}(\epsilon) \boldsymbol{P}_{1} & \boldsymbol{N}(\epsilon) \boldsymbol{P}_{2} & \boldsymbol{N}(\epsilon) \boldsymbol{P}_{3} & \boldsymbol{N}(\epsilon) \boldsymbol{P}_{4}
\end{array}\right]^{\mathrm{T}} \\
& =\boldsymbol{\Lambda}(\epsilon) \overline{\boldsymbol{P}}
\end{aligned}
$$

where $\boldsymbol{N}(\epsilon)=\left[\begin{array}{llll}N_{1, \mu}(\epsilon) & N_{2, \mu}(\epsilon) & \cdots & N_{n, \mu}(\epsilon)\end{array}\right]$ is the vector of B-spline basis functions and $\boldsymbol{\Lambda}(\epsilon)=\operatorname{diag}\{\boldsymbol{N}(\epsilon), \boldsymbol{N}(\epsilon), \boldsymbol{N}(\epsilon), \boldsymbol{N}(\epsilon)\} . \boldsymbol{P}_{j}=\left[\begin{array}{llll}P_{1, j} & P_{2, j} & \cdots & P_{n, j}\end{array}\right]^{\mathrm{T}}$ is the set of control points for $z_{j}$ where $j=1,2,3,4$ denotes the $j$ th element in $\boldsymbol{z}(t) . \overline{\boldsymbol{P}}=\left[\begin{array}{llll}\boldsymbol{P}_{1}^{\mathrm{T}} & \boldsymbol{P}_{2}^{\mathrm{T}} & \boldsymbol{P}_{3}^{\mathrm{T}} & \boldsymbol{P}_{4}^{\mathrm{T}}\end{array}\right]^{\mathrm{T}}$ is the control point vector treated as the decision variable in the online optimisation process. For a fixed time horizon $T, \epsilon \in[0,1]$ represents the normalised time index such that the conversion relationship between $\tau$ and $\epsilon$ is

$$
\tau=t+\epsilon T
$$

Thus, the first-order time derivatives of the basis functions are calculated as

$$
\frac{d N_{i, \mu}(\epsilon)}{d \tau}=\frac{d N_{i, \mu}(\epsilon)}{d \epsilon} \frac{d \epsilon}{d \tau}=\frac{1}{T} \frac{d N_{i, \mu}(\epsilon)}{d \epsilon}
$$

and the $r$-th time derivatives of the flat outputs $z_{j}(\tau), j=1,2,3,4$ can also be derived as

$$
z_{j}^{(r)}(\tau)=\frac{1}{T^{r}} \boldsymbol{N}^{(r)}(\epsilon) \boldsymbol{P}_{j} .
$$

By substituting Eqs. (26) and (29) into Eq. (21), the terminal cost, stage cost, and input cost terms can be rewritten as

$$
\begin{gathered}
\left\|\boldsymbol{z}(t+T)-\boldsymbol{z}_{\mathrm{r}}(t+T)\right\|_{\mathbf{Q}}^{2} \\
=\overline{\boldsymbol{P}}^{\mathrm{T}} \boldsymbol{\Lambda}^{\mathrm{T}}(1) \mathbf{Q} \boldsymbol{\Lambda}(1) \overline{\boldsymbol{P}}-2 \overline{\boldsymbol{P}}^{\mathrm{T}} \boldsymbol{\Lambda}^{\mathrm{T}}(1) \mathbf{Q} \boldsymbol{z}_{\mathrm{r}}(t+T)+\boldsymbol{z}_{\mathrm{r}}^{\mathrm{T}}(t+T) \mathbf{Q} \boldsymbol{z}_{\mathrm{r}}(t+T)^{\prime} \\
\left\|\boldsymbol{z}(\tau)-\boldsymbol{z}_{\mathrm{r}}(\tau)\right\|_{\mathbf{R}}^{2} \\
=\overline{\boldsymbol{P}}^{\mathrm{T}} \boldsymbol{\Lambda}^{\mathrm{T}}(\epsilon) \mathbf{R} \boldsymbol{\Lambda}(\epsilon) \overline{\boldsymbol{P}}-2 \overline{\boldsymbol{P}}^{\mathrm{T}} \boldsymbol{\Lambda}^{\mathrm{T}}(\epsilon) \mathbf{R} \boldsymbol{z}_{\mathrm{r}}(\tau)+\boldsymbol{z}_{\mathrm{r}}^{\mathrm{T}}(\tau) \mathbf{R} \boldsymbol{z}_{\mathrm{r}}(\tau)
\end{gathered}
$$

and

$$
\begin{aligned}
& \|\ddot{\boldsymbol{z}}(\tau)\|_{\mathbf{S}}^{2} \\
= & \overline{\boldsymbol{P}}^{\mathrm{T}} \ddot{\boldsymbol{\Lambda}}^{\mathrm{T}}(\epsilon) \mathbf{S} \ddot{\boldsymbol{\Lambda}}(\epsilon) \overline{\boldsymbol{P}}
\end{aligned}
$$


where $\epsilon=1$ in Eq. (30) is obtained from the conversion relationship (27) for choosing $\epsilon=1$ obtains $\tau=t+T$ from $\tau=t+\epsilon T . \ddot{\boldsymbol{\Lambda}}(\epsilon)=\operatorname{diag}\left\{\frac{1}{T^{2}} \ddot{\boldsymbol{N}}(\epsilon), \frac{1}{T^{2}} \ddot{\boldsymbol{N}}(\epsilon), \frac{1}{T^{2}} \ddot{\boldsymbol{N}}(\epsilon), \frac{1}{T^{2}} \ddot{\boldsymbol{N}}(\epsilon)\right\}$ denotes the matrix of the second-order derivatives of basis functions. Furthermore, by defining

$$
\begin{gathered}
\mathbf{Q}_{\mathrm{t}}=\int_{t}^{t+T}\left(\boldsymbol{\Lambda}^{\mathrm{T}}(\epsilon) \mathbf{R} \boldsymbol{\Lambda}(\epsilon)+\ddot{\boldsymbol{\Lambda}}^{\mathrm{T}}(\epsilon) \mathbf{S} \ddot{\boldsymbol{\Lambda}}(\epsilon)\right) d \tau, \\
\boldsymbol{G}_{\mathrm{t}}=-2 \int_{t}^{t+T} \boldsymbol{\Lambda}^{\mathrm{T}}(\epsilon) \mathbf{R} \boldsymbol{z}_{\mathrm{r}}(\tau) d \tau, \\
\mathbf{Q}_{1}=\boldsymbol{\Lambda}^{\mathrm{T}}(1) \mathbf{Q} \boldsymbol{\Lambda}(1), \\
\boldsymbol{G}_{1}=-2 \boldsymbol{\Lambda}^{\mathrm{T}}(1) \mathbf{Q} \boldsymbol{z}_{\mathrm{r}}(t+T),
\end{gathered}
$$

and

$$
C=\boldsymbol{z}_{\mathrm{r}}^{\mathrm{T}}(t+T) \mathbf{Q} \boldsymbol{z}_{\mathrm{r}}(t+T)+\int_{t}^{t+T} \boldsymbol{z}_{\mathrm{r}}^{\mathrm{T}}(\tau) \mathbf{R} \boldsymbol{z}_{\mathrm{r}}(\tau) d \tau
$$

the cost function (13) can be expressed in the following compact form

$$
J(\overline{\boldsymbol{P}})=\overline{\boldsymbol{P}}^{\mathrm{T}} \overline{\mathbf{Q}} \overline{\boldsymbol{P}}+\overline{\boldsymbol{P}}^{\mathrm{T}} \overline{\boldsymbol{G}}+C
$$

where $\overline{\mathbf{Q}}=\mathbf{Q}_{\mathrm{t}}+\mathrm{Q}_{1}$ and $\overline{\boldsymbol{G}}=\boldsymbol{G}_{\mathrm{t}}+\boldsymbol{G}_{1}$.

Regarding the initial condition (23a), it should be satisfied to ensure that the optimised trajectory can start smoothly from the current vehicle states. According to the derivatives of the B-spline curve at the endpoint, the first three control points $P_{1, j}, P_{2, j}$, and $P_{3, j}$ of each flat output element can be determined as:

$$
\begin{aligned}
& P_{1, j}=z_{j}(0) \\
& P_{2, j}=\frac{\ddot{z}_{j}(0) \epsilon_{\mu+2}}{\mu} T+P_{1, j} \\
& P_{3, j}=\frac{\ddot{z}_{j}(0) \epsilon_{\mu+2} \epsilon_{\mu+3}}{(\mu-1) \mu} T^{2}+\frac{\epsilon_{\mu+2}+\epsilon_{\mu+3}}{\epsilon_{\mu+2}} P_{2, j}-\frac{\epsilon_{\mu+3}}{\epsilon_{\mu+2}} P_{1, j}
\end{aligned}
$$

where $z_{j}(0), \dot{z}_{j}(0)$ and $\ddot{z}_{j}(0)$ are the current position, velocity and acceleration provided by corresponding sensors and the parameters $\epsilon_{\mu+2}$ and $\epsilon_{\mu+3}$ are the knots defined in (25). Since this relationship can further scale down the dimension of the control point vector by three, the actual number of variables need to be optimised in the online optimisation is $j(n-3)$.

Finally, the optimal control problem (22) and (23) is simplified as

$$
\min _{\overline{\boldsymbol{P}}} \overline{\boldsymbol{P}}^{\mathrm{T}} \overline{\mathbf{Q}} \overline{\boldsymbol{P}}+\overline{\boldsymbol{P}}^{\mathrm{T}} \overline{\boldsymbol{G}}
$$

subject to

$$
\begin{gathered}
\mathbf{L}_{\text {eq }} \overline{\boldsymbol{P}}=\boldsymbol{W}_{\text {eq }} \\
\mathbf{L}_{\text {ineq }} \overline{\boldsymbol{P}} \leq \boldsymbol{W}_{\text {ineq }}
\end{gathered}
$$


where the constraints (36) are the parametrised linear form of the initial condition (34) and inequality constraint (23b). Through all these transformations, the original optimal control problem become tractable because it has been formulated into a standard QP problem which can be conveniently solved by using the efficient QP solvers. This process will significantly relieve the computational burden of solving the online optimal control problem.

The feasibility of this optimal control problem (35) and (36) (i.e. the existence of an admissible solution) is discussed in Faulwasser et al. (2014). Given the optimised solution $\overline{\boldsymbol{P}}^{*}$, the time evolution of the optimal state $\boldsymbol{x}^{*}$ and control input $\boldsymbol{u}^{*}$ can be calculated by reversely using the B-spline parametrisation (26) and the differential flatness properties (17) and (18). Even though the computational demand of the online optimal control is reduced dramatically, it is still too severe to achieve the real-time closed-loop flight control for the quadrotor. Moreover, the multiple disturbances may heavily degrade the performance of this kind of optimal control. Therefore, $\boldsymbol{u}^{*}$ can not be directly used to control the quadrotor. However, it is logical to let $\boldsymbol{x}^{*}$ and $\boldsymbol{u}^{*}$ act as the reference pair $\left(\boldsymbol{x}_{\text {ref }}, \boldsymbol{u}_{\text {ref }}\right)$ for an inner-loop robust linear controller, as shown in (11), to accomplish the asymptotical trajectory tracking purpose.

\section{Composite hierarchical anti-disturbance control design}

For the linearised error system (12) with unknown lumped disturbance $\boldsymbol{d}$ and linearisation error $\Delta f$, an anti-disturbance controller is constructed to guarantee the robustness of closed-loop system. Note that the error variables $\tilde{\boldsymbol{x}}_{\mathrm{e}}$ and $\tilde{\boldsymbol{u}}_{\mathrm{fb}}$ are replaced by $\boldsymbol{x}$ and $\boldsymbol{u}$ with a slight abuse of the notations, in order to simplify the deviation in this section. Thus, Eq. (12) is rewritten as

$$
\dot{\boldsymbol{x}}=\mathbf{A} \boldsymbol{x}+\mathbf{B} \boldsymbol{u}+\mathbf{B}_{\mathrm{d}} \boldsymbol{d}+\Delta f(\boldsymbol{x}, \boldsymbol{u}) .
$$

To estimate the lumped disturbance $\boldsymbol{d}$ in Eq. (37), a linear disturbance observer is designed as

$$
\left\{\begin{array}{l}
\hat{d}=\boldsymbol{\xi}+\mathbf{L} \boldsymbol{x} \\
\dot{\boldsymbol{\xi}}=-\mathbf{L B}_{\mathrm{d}}(\boldsymbol{\xi}+\mathbf{L} \boldsymbol{x})-\mathbf{L}(\mathbf{A} \boldsymbol{x}+\mathbf{B} \boldsymbol{u})
\end{array} .\right.
$$

The the reduced-order observer dose not directly estimate the disturbance but introduces an internal state with carefully designed dynamics in the disturbance estimation process (Chen, Ballance, Gawthrop, \& John, 2000). $\hat{\boldsymbol{d}}=\left[\begin{array}{llllll}\hat{d}_{x} & \hat{d}_{y} & \hat{d}_{z} & \hat{d}_{\phi} & \hat{d}_{\theta} & \hat{d}_{\psi}\end{array}\right]^{\mathrm{T}}$ is the estimate vector of $\boldsymbol{d}$, $\mathbf{L}$ is the disturbance observer gain matrix, and $\boldsymbol{\xi}$ is the internal state vector of the disturbance observer. Define $\tilde{\boldsymbol{d}}=\boldsymbol{d}-\hat{\boldsymbol{d}}$ as the estimation error vector, where $\tilde{\boldsymbol{d}}=\left[\begin{array}{llllll}\tilde{d}_{x} & \tilde{d}_{y} & \tilde{d}_{z} & \tilde{d}_{\phi} & \tilde{d}_{\theta} & \tilde{d}_{\psi}\end{array}\right]^{\mathrm{T}}$. Taking the time derivative of $\tilde{\boldsymbol{d}}$ yields the estimation error dynamics:

$$
\dot{\tilde{d}}=\dot{\boldsymbol{d}}-\mathbf{L} \mathbf{B}_{\mathrm{d}} \tilde{\boldsymbol{d}}-\mathbf{L} \Delta f
$$

The lumped disturbance $\boldsymbol{d}$ can be divided into matched disturbance $\boldsymbol{d}_{1}=\left[\begin{array}{llll}d_{z} & d_{\phi} & d_{\theta} & d_{\psi}\end{array}\right]^{\mathrm{T}}$ and mismatched disturbance $\boldsymbol{d}_{2}=\left[\begin{array}{ll}d_{x} & d_{y}\end{array}\right]^{\mathrm{T}}$ where mismatched disturbance means that the disturbance enters the system via a different path from the control input (Yang, Zolotas, Chen, Michail, \& Li, 2011). In the absence of the linearisation error $\Delta f$, the mismatched disturbances $d_{x}$ and $d_{y}$ can be partitioned from model (37) into the following subsystems:

$$
\left\{\begin{array} { l } 
{ \ddot { x } = - g \theta + d _ { x } } \\
{ \dot { \theta } = \dot { \theta } } \\
{ \ddot { \theta } = u _ { \theta } + d _ { \theta } }
\end{array} \text { and } \left\{\begin{array}{l}
\ddot{y}=g \phi+d_{y} \\
\dot{\phi}=\dot{\phi} \\
\ddot{\phi}=u_{\phi}+d_{\phi}
\end{array} .\right.\right.
$$


Taking the mismatched disturbance $d_{x}$ as an example, in order to reject it, the state $\theta$ can be treated as an intermediate variable. By defining $\theta_{\mathrm{n}}=\theta-\frac{1}{g} \hat{d}_{x}$ as a new state variable to replace $\theta$, the corresponding subsystem in (40) is transformed to

$$
\left\{\begin{array}{l}
\ddot{x}=-g \theta_{\mathrm{n}}+\tilde{d}_{x} \\
\dot{\theta}_{\mathrm{n}}=\dot{\theta}_{\mathrm{n}} \\
\ddot{\theta}_{\mathrm{n}}=u_{\theta}+d_{\theta}-\frac{1}{g} \ddot{\hat{d_{x}}}
\end{array} .\right.
$$

where the remaining $\tilde{d}_{x}$ is the estimation error after disturbance compensation. To guarantee $\theta_{\mathrm{n}} \rightarrow 0$ as $t \rightarrow \infty, \theta$ needs to be stabilised at the new operating point $\frac{1}{g} \hat{d}_{x}$ rather than 0 . That is to say, the mismatched disturbance is compensated through coordinate transformation (Peng et al., 2015). Similarly, mismatched disturbance $d_{y}$ also can be rejected by choosing $\phi_{\mathrm{n}}=\phi+\frac{1}{g} \hat{d}_{y}$. The resulting subsystem is derived as

$$
\left\{\begin{array}{l}
\ddot{y}=g \phi_{\mathrm{n}}+\tilde{d}_{y} \\
\dot{\phi}_{\mathrm{n}}=\dot{\phi}_{\mathrm{n}} \\
\ddot{\phi}_{\mathrm{n}}=u_{\phi}+d_{\phi}+\frac{1}{g} \ddot{\hat{d}}_{y}
\end{array} .\right.
$$

Using subsystems (41) and (42) to replace the their counterparts (40) in (37), the new system state vector becomes $\boldsymbol{x}_{\mathrm{n}}=\left[\begin{array}{llllllllllll}x & y & z & \dot{x} & \dot{y} & \dot{z} & \phi_{\mathrm{n}} & \theta_{\mathrm{n}} & \psi & \dot{\phi}_{\mathrm{n}} & \dot{\theta}_{\mathrm{n}} & \dot{\psi}\end{array}\right]^{\mathrm{T}}$ which contains the mismatched disturbance estimate $\hat{\boldsymbol{d}}_{2}=\left[\begin{array}{ll}\hat{d}_{x} & \hat{d}_{y}\end{array}\right]^{\mathrm{T}}$. Consequently, the overall linear model can be rewritten as

$$
\dot{\boldsymbol{x}}_{\mathrm{n}}=\mathbf{A} \boldsymbol{x}_{\mathrm{n}}+\mathbf{B} \boldsymbol{u}+\Delta f+\mathbf{B}_{1} \boldsymbol{d}_{1}+\mathbf{B}_{2} \tilde{\boldsymbol{d}}_{2}+\mathbf{B}_{3} \ddot{\hat{\boldsymbol{d}}}_{2}
$$

where the coefficient matrices $\mathbf{B}_{1}, \mathbf{B}_{2}$ and $\mathbf{B}_{3}$ are given as follows

$$
\mathbf{B}_{1}=\left[\begin{array}{c|c}
\mathbf{0}_{3 \times 1} & \mathbf{0}_{3 \times 3} \\
\hline 0 & \mathbf{0}_{1 \times 3} \\
0 & \mathbf{0}_{1 \times 3} \\
1 & \mathbf{0}_{1 \times 3} \\
\hline \mathbf{0}_{3 \times 1} & \mathbf{0}_{3 \times 3} \\
\mathbf{0}_{3 \times 1} & \mathbf{I}_{3 \times 3}
\end{array}\right], \mathbf{B}_{2}=\left[\begin{array}{cc}
\mathbf{0}_{3 \times 2} \\
\hline 1 & 0 \\
0 & 1 \\
0 & 0 \\
\hline \mathbf{0}_{3 \times 2} \\
\mathbf{0}_{3 \times 2}
\end{array}\right] \text {, and } \mathbf{B}_{3}=\left[\begin{array}{cc}
\mathbf{0}_{3 \times 2} \\
\hline-\frac{1}{g} & 0 \\
0 & \frac{1}{g} \\
0 & 0 \\
\hline \mathbf{0}_{3 \times 2} \\
\mathbf{0}_{3 \times 2}
\end{array}\right]
$$

The mismatched disturbance $\boldsymbol{d}_{2}$ is compensated through coordinate shift, whereas the matched disturbance $\boldsymbol{d}_{1}$ can be directly rejected by the following composite control law:

$$
\boldsymbol{u}=\mathbf{K} \boldsymbol{x}_{\mathrm{n}}-\mathbf{B}_{u} \hat{\boldsymbol{d}}_{1}
$$

where $\boldsymbol{u}_{\text {nom }} \triangleq \mathbf{K} \boldsymbol{x}_{\mathrm{n}}$ is the nominal control input; the coefficient matrix $\mathbf{B}_{u}$ is derived from the relationship $\mathbf{B B}_{u}=\mathbf{B}_{1}$ such that

$$
\mathbf{B}_{u}=\left[\begin{array}{c|c}
-m & \mathbf{0}_{1 \times 3} \\
\hline \mathbf{0}_{3 \times 1} & \mathbf{I}_{3 \times 3}
\end{array}\right]
$$


Substituting Eq. (44) into Eq. (43) yields the following closed-loop system

$$
\begin{aligned}
\dot{\boldsymbol{x}}_{\mathrm{n}} & =\mathbf{A} \boldsymbol{x}_{\mathrm{n}}+\mathbf{B K} \boldsymbol{x}_{\mathrm{n}}-\mathbf{B B}_{u} \hat{\boldsymbol{d}}_{1}+\mathbf{B}_{1} \boldsymbol{d}_{1}+\mathbf{B}_{2} \tilde{\boldsymbol{d}}_{2}+\Delta f+\mathbf{B}_{3} \ddot{\hat{\boldsymbol{d}}}_{2} . \\
& =(\mathbf{A}+\mathbf{B K}) \boldsymbol{x}_{\mathrm{n}}+\mathbf{B}_{d} \tilde{\boldsymbol{d}}+\Delta f+\mathbf{B}_{3} \ddot{\hat{\boldsymbol{d}}}_{2}
\end{aligned}
$$

Combining the (39) and (45), the closed-loop augmented system is given by

$$
\underbrace{\left[\begin{array}{c}
\dot{\boldsymbol{x}}_{\mathrm{n}} \\
\dot{\tilde{\boldsymbol{d}}}
\end{array}\right]}_{\overline{\bar{x}}}=\underbrace{\left[\begin{array}{cc}
\mathbf{A}+\mathbf{B K} & \mathbf{B}_{\mathrm{d}} \\
\mathbf{0} & -\mathbf{L B}_{\mathrm{d}}
\end{array}\right]}_{\overline{\mathbf{A}}} \underbrace{\left[\begin{array}{c}
\boldsymbol{x}_{\mathrm{n}} \\
\tilde{\boldsymbol{d}}
\end{array}\right]}_{\overline{\boldsymbol{x}}}+\underbrace{\left[\begin{array}{c}
\mathbf{I} \\
-\mathbf{L}
\end{array}\right]}_{\mathbf{F}} \Delta f+\underbrace{\left[\begin{array}{cc}
\mathbf{B}_{3} & \mathbf{0} \\
\mathbf{0} & \mathbf{I}
\end{array}\right]}_{\overline{\mathbf{B}}} \underbrace{\left[\begin{array}{c}
\ddot{\hat{\boldsymbol{d}}}_{2} \\
\dot{\boldsymbol{d}}
\end{array}\right]}_{\overline{\boldsymbol{d}}},
$$

or compactly,

$$
\dot{\overline{\boldsymbol{x}}}=\overline{\mathbf{A}} \overline{\boldsymbol{x}}+\mathbf{F} \Delta f+\overline{\mathbf{B}} \overline{\boldsymbol{d}} .
$$

The flat output vector $\boldsymbol{z}=\left[\begin{array}{llll}x & y & z & \psi\end{array}\right]^{\mathrm{T}}$ can be picked from the augmented state $\overline{\boldsymbol{x}}$ as

$$
\boldsymbol{z}=\underbrace{\left[\begin{array}{ll}
\mathbf{C} & \mathbf{0}
\end{array}\right]}_{\boldsymbol{C}_{\mathrm{m}}} \overline{\boldsymbol{x}} .
$$

As for the linearisation error $\Delta f(\overline{\boldsymbol{x}})$, based on Assumption 1, it is assumed that $\|\Delta f(\overline{\boldsymbol{x}})\| \leq\left\|\mathbf{U}_{\mathrm{m}} \overline{\boldsymbol{x}}\right\|$ where $\mathbf{U}_{\mathrm{m}}=\left[\begin{array}{ll}\mathbf{U} & \mathbf{0}\end{array}\right]$.

Assumption 2: The lumped disturbance $\boldsymbol{d}$ is slowly time varying with bounded variation such that $\dot{\boldsymbol{d}} \in L_{2}[0, \infty)$ and $\ddot{\hat{\boldsymbol{d}}}_{2} \in L_{2}[0, \infty)$ can be deduced, which means $\overline{\boldsymbol{d}} \in L_{2}[0, \infty)$.

To attenuate the remaining disturbance $\overline{\boldsymbol{d}}, H_{\infty}$ performance index is integrated in the feedback controller design to improve the robustness against this norm-bounded disturbance. Under the $H_{\infty}$ controller, the following performance inequality is satisfied

$$
\|\boldsymbol{z}(\tau)\|^{2}<\gamma^{2}\|\overline{\boldsymbol{d}}(\tau)\|^{2} .
$$

In the following, a sufficient condition is given, under which the closed-loop system is asymptotically stable and satisfies the $H_{\infty}$ disturbance attenuation performance. The observer gain $L$ and control gain $K$ are designed during the proof process.

Theorem 5.1: Consider system (37) with disturbance and linearisation error under Assumption 1 and Assumption 2. Given parameters $\lambda>0$ and $\gamma>0$, if there exist $\mathbf{V}_{1}>0, \mathbf{W}_{2}>0, \mathbf{Y}_{1}$, and $\mathbf{Y}_{2}$ satisfying

$$
\left[\begin{array}{ccccccc}
\mathbf{\Pi}_{1} & \mathbf{B}_{\mathrm{d}} & \mathbf{I} & \mathbf{B}_{3} & \mathbf{0} & \mathbf{V}_{1}^{\mathrm{T}} \mathbf{U}^{\mathrm{T}} & \mathbf{V}_{1}^{\mathrm{T}} \mathbf{C}^{\mathrm{T}} \\
* & \mathbf{\Pi}_{2} & -\mathbf{Y}_{2} & \mathbf{0} & \mathbf{W}_{2} & \mathbf{0} & \mathbf{0} \\
* & * & -\frac{1}{\lambda^{2}} \mathbf{I} & \mathbf{0} & \mathbf{0} & \mathbf{0} & \mathbf{0} \\
* & * & * & -\gamma^{2} \mathbf{I} & \mathbf{0} & \mathbf{0} & \mathbf{0} \\
* & * & * & * & -\gamma^{2} \mathbf{I} & \mathbf{0} & \mathbf{0} \\
* & * & * & * & * & -\lambda^{2} \mathbf{I} & \mathbf{0} \\
* & * & * & * & * & * & -\mathbf{I}
\end{array}\right]<\mathbf{0}
$$


where

$$
\begin{aligned}
& \mathbf{\Pi}_{1}=\mathbf{V}_{1}^{\mathrm{T}} \mathbf{A}^{\mathrm{T}}+\mathbf{A} \mathbf{V}_{1}+\mathbf{Y}_{1}^{\mathrm{T}} \mathbf{B}^{\mathrm{T}}+\mathbf{B} \mathbf{Y}_{1} \\
& \boldsymbol{\Pi}_{2}=-\mathbf{B}_{\mathrm{d}}^{\mathrm{T}} \mathbf{Y}_{2}^{\mathrm{T}}-\mathbf{Y}_{2} \mathbf{B}_{\mathrm{d}}
\end{aligned}
$$

then the closed-loop augmented system in (47) and (48) under the composite anti-disturbance control law (44) is asymptotically stable and satisfies $\|\boldsymbol{z}(\tau)\|^{2}<\gamma^{2}\|\overline{\boldsymbol{d}}(\tau)\|^{2}$. The controller gain is given by $\mathbf{K}=\mathbf{Y}_{1} \mathbf{V}_{1}^{-1}$ and the gain of disturbance observer (38) is given by $\mathbf{L}=\mathbf{W}_{2}^{-1} \mathbf{Y}_{2}$.

Proof. Define a Lyapunov functional candidate as

$$
V(\overline{\boldsymbol{x}}(t))=\overline{\boldsymbol{x}}^{\mathrm{T}}(t) \mathbf{W} \overline{\boldsymbol{x}}(t)+\frac{1}{\lambda^{2}} \int_{0}^{t}\left(\left\|\mathbf{U}_{\mathrm{m}} \overline{\boldsymbol{x}}(\tau)\right\|^{2}-\|\Delta f(\overline{\boldsymbol{x}}(\tau))\|^{2}\right) d \tau .
$$

where the integral item is derived from Assumption 1. The parameter $\lambda$ brings an additional degree of freedom when solving the linear matrix inequalities (LMIs), which can reduce the conservatism of the Lyapunov function. Differentiating $V(\overline{\boldsymbol{x}}(t))$ along with the trajectories of $(47)$ in the absence of $\overline{\boldsymbol{d}}$ yields

$$
\begin{aligned}
\dot{V} & =\operatorname{sym}\left(\overline{\boldsymbol{x}}^{\mathrm{T}} \mathbf{W}(\overline{\mathbf{A}} \overline{\boldsymbol{x}}+\mathbf{F} \Delta f)\right)+\frac{1}{\lambda^{2}}\left(\overline{\boldsymbol{x}}^{\mathrm{T}} \mathbf{U}_{\mathrm{m}}^{\mathrm{T}} \mathbf{U}_{\mathrm{m}} \overline{\boldsymbol{x}}-\Delta f^{\mathrm{T}} \Delta f\right) \\
& =\left[\begin{array}{c}
\overline{\boldsymbol{x}} \\
\Delta f
\end{array}\right]^{\mathrm{T}}\left[\begin{array}{cc}
\overline{\mathbf{A}}^{\mathrm{T}} \mathbf{W}+\mathbf{W} \overline{\mathbf{A}}+\frac{1}{\lambda^{2}} \mathbf{U}_{\mathrm{m}}^{\mathrm{T}} \mathbf{U}_{\mathrm{m}} & \mathbf{W} \mathbf{F} \\
\mathbf{F}^{\mathrm{T}} \mathbf{W} & -\frac{1}{\lambda^{2}} \mathbf{I}
\end{array}\right]\left[\begin{array}{c}
\overline{\boldsymbol{x}} \\
\Delta f
\end{array}\right] . \\
& =\boldsymbol{\xi}^{\mathrm{T}} \boldsymbol{\Xi}_{1} \boldsymbol{\xi}
\end{aligned}
$$

For the sake of simplicity, we denote the notation $\operatorname{sym}(\boldsymbol{N})=\boldsymbol{N}+\boldsymbol{N}^{\mathrm{T}}$. According to the Lyapunov theory, if $\boldsymbol{\Xi}_{1}<0$ holds, the closed-loop augmented system (47) is asymptotically stable.

The next step is to verify the disturbance attenuation performance index (49). Consider the following function:

$$
\begin{aligned}
S(t) & =\dot{V}(\overline{\boldsymbol{x}}(t))+\|\boldsymbol{z}(t)\|^{2}-\gamma^{2}\|\overline{\boldsymbol{d}}(t)\|^{2} \\
& =\operatorname{sym}\left(\overline{\boldsymbol{x}}^{\mathrm{T}} \mathbf{W}(\overline{\mathbf{A}} \overline{\boldsymbol{x}}+\mathbf{F} \Delta f+\overline{\mathbf{B}} \overline{\boldsymbol{d}})\right)+\frac{1}{\lambda^{2}}\left(\overline{\boldsymbol{x}}^{\mathrm{T}} \mathbf{U}_{\mathrm{m}}^{\mathrm{T}} \mathbf{U}_{\mathrm{m}} \overline{\boldsymbol{x}}-\Delta f^{\mathrm{T}} \Delta f\right)+\left(\overline{\boldsymbol{x}}^{\mathrm{T}} \mathbf{C}_{\mathrm{m}}^{\mathrm{T}} \mathbf{C}_{\mathrm{m}} \overline{\boldsymbol{x}}-\gamma^{2} \overline{\boldsymbol{d}}^{\mathrm{T}} \overline{\boldsymbol{d}}\right) \\
& =\left[\begin{array}{c}
\overline{\boldsymbol{x}} \\
\Delta f \\
\overline{\boldsymbol{d}}
\end{array}\right]^{\mathrm{T}}\left[\begin{array}{ccc}
\overline{\mathbf{A}}^{\mathrm{T}} \mathbf{W}+\mathbf{W} \overline{\mathbf{A}}+\frac{1}{\lambda^{2}} \mathbf{U}_{\mathrm{m}}^{\mathrm{T}} \mathbf{U}_{\mathrm{m}}+\mathbf{C}_{\mathrm{m}}^{\mathrm{T}} \mathbf{C}_{\mathrm{m}} & \mathbf{W F} & \mathbf{W} \overline{\mathbf{B}} \\
* & -\frac{1}{\lambda^{2}} \mathbf{I} & \mathbf{0} \\
* & * & -\gamma^{2} \mathbf{I}
\end{array}\right]\left[\begin{array}{c}
\overline{\boldsymbol{x}} \\
\Delta f \\
\overline{\boldsymbol{d}}
\end{array}\right] . \\
& =\overline{\boldsymbol{\xi}}^{\mathrm{T}} \boldsymbol{\Xi}_{2} \overline{\boldsymbol{\xi}}
\end{aligned}
$$

Define $J(t)=\int_{0}^{t} S(\tau) d \tau$. Under the zero initial condition, we obtain the following equation:

$$
J(t)=V(\overline{\boldsymbol{x}}(t))+\int_{0}^{t}\left(\|\boldsymbol{z}(\tau)\|^{2}-\gamma^{2}\|\overline{\boldsymbol{d}}(\tau)\|^{2}\right) d \tau .
$$

It can be observed that if $\boldsymbol{\Xi}_{2}<0$ holds, $S(t)<0$ and $J(t)<0$ are obvious, which further leads to the inequality $\|\boldsymbol{z}(\tau)\|^{2}<\gamma^{2}\|\overline{\boldsymbol{d}}(\tau)\|^{2}$. Moreover, $\boldsymbol{\Xi}_{2}<0$ also implies $\boldsymbol{\Xi}_{1}<0$ by using the Schur complement such that the closed-loop augmented system is asymptotically stable. 
Now we begin to verify that if $\boldsymbol{\Xi}_{2}<0$, then (50) holds. Letting

$$
\mathbf{W}=\left[\begin{array}{cc}
\mathbf{W}_{1} & \mathbf{0} \\
\mathbf{0} & \mathbf{W}_{2}
\end{array}\right]=\left[\begin{array}{cc}
\mathbf{V}_{1}^{-1} & \mathbf{0} \\
\mathbf{0} & \mathbf{W}_{2}
\end{array}\right]>\mathbf{0}
$$

and substituting $\mathbf{W}$ into $\boldsymbol{\Xi}_{2}<\mathbf{0}$, it becomes

$$
\boldsymbol{\Xi}_{3}=\left[\begin{array}{ccccccc}
\boldsymbol{\Gamma}_{1} & \mathbf{W}_{1} \mathbf{B}_{\mathrm{d}} & \mathbf{W}_{1} & \mathbf{W}_{1} \mathbf{B}_{3} & \mathbf{0} & \mathbf{U}^{\mathrm{T}} & \mathbf{C}^{\mathrm{T}} \\
* & \boldsymbol{\Gamma}_{2} & -\mathbf{W}_{2} \mathbf{L} & \mathbf{0} & \mathbf{W}_{2} & \mathbf{0} & \mathbf{0} \\
* & * & -\frac{1}{\lambda^{2}} \mathbf{I} & \mathbf{0} & \mathbf{0} & \mathbf{0} & \mathbf{0} \\
* & * & * & -\gamma^{2} \mathbf{I} & \mathbf{0} & \mathbf{0} & \mathbf{0} \\
* & * & * & * & -\gamma^{2} \mathbf{I} & \mathbf{0} & \mathbf{0} \\
* & * & * & * & * & -\lambda^{2} \mathbf{I} & \mathbf{0} \\
* & * & * & * & * & * & -\mathbf{I}
\end{array}\right]<\mathbf{0}
$$

where

$$
\begin{aligned}
& \boldsymbol{\Gamma}_{1}=\mathbf{A}^{\mathrm{T}} \mathbf{W}_{1}+\mathbf{W}_{1} \mathbf{A}+\mathbf{K}^{\mathrm{T}} \mathbf{B}^{\mathrm{T}} \mathbf{W}_{1}+\mathbf{W}_{1} \mathbf{B K} \\
& \boldsymbol{\Gamma}_{2}=-\mathbf{B}_{\mathrm{d}}^{\mathrm{T}} \mathbf{L}^{\mathrm{T}} \mathbf{W}_{2}-\mathbf{W}_{2} \mathbf{L} \mathbf{B}_{\mathrm{d}}
\end{aligned}
$$

Then pre-multiplying and post-multiplying $\operatorname{diag}\left\{\mathbf{V}_{1}, \mathbf{I}, \mathbf{I}, \mathbf{I}, \mathbf{I}, \mathbf{I}, \mathbf{I}\right\}$ to the left and right sides of $\boldsymbol{\Xi}_{3}$ and denoting $\mathbf{Y}_{1}=\mathbf{K} \mathbf{V}_{1}$ and $\mathbf{Y}_{2}=\mathbf{W}_{2} \mathbf{L}$, we can obtain (50) and the corresponding conclusion.

As mentioned in the introduction, the composite hierarchical anti-disturbance control strategy first introduces a disturbance observer to estimate the lumped disturbance $\boldsymbol{d}$. Then, based on the estimate $\hat{\boldsymbol{d}}$, the matched and mismatched disturbances are separately compensated through direct rejection and coordinate shift in the inner layer. After that, the remaining portion $\overline{\boldsymbol{d}}$ is further attenuated by employing an outer layer $H_{\infty}$ controller. This hierarchical control scheme provides an efficient way to improve the robustness against disturbance.

By summarising the sections 3,4 , and 5 , an overall diagram of the proposed constrained antidisturbance control strategy is depicted in Fig. 2.

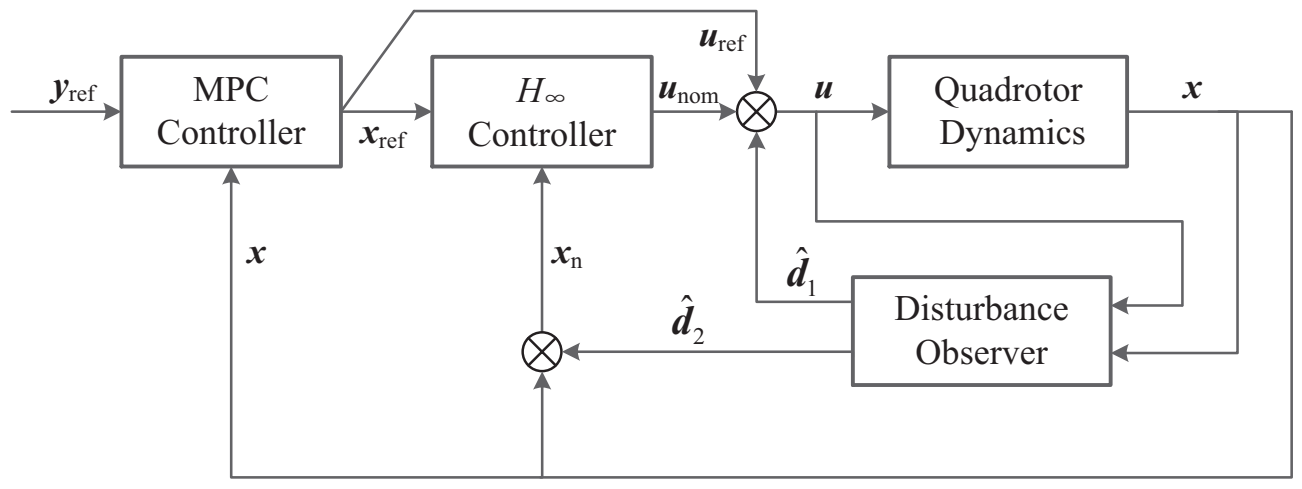

Figure 2. Control block diagram

Remark 3: The proposed anti-disturbance control method is based on the condition that all the state variables can be measured. When there are missing measurements and random disturbances, the state estimation problems with variance-constraints become concern. The new methods reported in Hu, Wang, Shen, \& Gao (2013) and Hu, Wang, Liu, \& Gao (2016) would be helpful for further extensions. 


\section{Simulation studies}

This section includes two case studies to demonstrate the performance of the proposed constrained anti-disturbance control. By carrying out the simulations, the capability of handling the constraints on states and inputs is first verified and then the robust performance against disturbances is tested. The simulations are implemented in the Simulink environment and the qpOASES toolbox is employed as the QP solver to solve the quadratic programming problem (35) and (36). The model parameters of the quadrotor used in the simulations are given in the Table 1. In the MPC

Table 1. Quadrotor parameters

\begin{tabular}{lclc}
\hline Notation & Value & Notation & Value \\
\hline $\mathrm{m}, \mathrm{kg}$ & 2 & $\rho, \mathrm{N} / \mathrm{rpm}^{2}$ & $3 \times 10^{-6}$ \\
$\mathrm{~J}, \mathrm{~kg} \cdot \mathrm{m}^{2}$ & $\operatorname{diag}\left\{5 \times 10^{-3}, 5 \times 10^{-3}, 9 \times 10^{-3}\right\}$ & $\kappa, \mathrm{N} \cdot \mathrm{m} / \mathrm{rpm}^{2}$ & $1.5 \times 10^{-7}$ \\
$\mathrm{l}, \mathrm{m}$ & 0.22 & & \\
\hline
\end{tabular}

design, the prediction horizon is set to $T=6 \mathrm{~s}$, the sampling interval is selected as $\delta=1 \mathrm{~s}$, and the weighting matrices in cost function (13) are chosen as unit diagonal matrices with appropriate dimensions. The 7th-order B-spline polynomials are used to parametrise the nonlinear optimisation into a simple QP problem. The knot vector which the B-spline basis functions are defined on is chosen as $\boldsymbol{U}_{\text {knot }} \triangleq\left[\begin{array}{llll}\mathbf{0}_{1 \times 8} & 0.3 & 0.6 & \mathbf{1}_{1 \times 8}\end{array}\right]$ and 10 control points are needed to determine each flat output element. For the linear anti-disturbance controller, according to the Theorem 5.1, the following controller gain $K$ and observer gain $L$ can be obtained:

$$
K=\left[\begin{array}{cccccccccccc}
0 & 0 & 8.66 & 0 & 0 & 3.76 & 0 & 0 & 0 & 0 & 0 & 0 \\
0 & -66.5 & 0 & 0 & -44.5 & 0 & -154 & 0 & 0 & -15.4 & 0 & 0 \\
66.5 & 0 & 0 & 44.5 & 0 & 0 & 0 & -154 & 0 & 0 & -15.4 & 0 \\
0 & 0 & 0 & 0 & 0 & 0 & 0 & 0 & -15.8 & 0 & 0 & -2.37
\end{array}\right]
$$

$$
L=\left[\begin{array}{c|ccc|c|ccc} 
& 0.8690 & 0 & 0 & & 0 & 0.0464 & 0 \\
& 0 & 0.8690 & 0 \\
\mathbf{0}_{6 \times 3} & 0 & 0 & 0.8585 & \mathbf{0}_{6 \times 3} & -0.0464 & 0 & 0 \\
& 0 & -0.0024 & 0 & & 0 & 0 \\
& 0.0024 & 0 & 0 & & 0 & 0 \\
& 0 & 0 & 0 & & 0 & 0 & 4.0208
\end{array}\right]
$$

where $\gamma=0.8, \lambda=2$, and the weighting matrix $\mathbf{U}=\operatorname{diag}\left\{\mathbf{0}_{1 \times 3}, \mathbf{0 . 8}_{1 \times 3}, \mathbf{0}_{1 \times 3}, \mathbf{0}_{1 \times 3}\right\}$ are used in solving the LMIs in the Theorem 5.1.

\subsection{Aggressive manoeuvre}

In this part, an abrupt step is commanded on longitudinal position to stimulate the quadrotor to its maximum pitch angle. First, a $20 \mathrm{~m}$ step is commanded, the pitch angle command generated by the outer-loop MPC and the actual pitch angle are shown in Fig. 3. It can seen that the pitch angle response reaches its lower bound. In the second test, the position step is increased to $25 \mathrm{~m}$ and the corresponding pitch angle response is depicted in Fig. 4. Compared to the Fig. 3, it can be observed that although the upper bound is also reached, the pitch angle response is well maintained within the lower bound. The position responses of the both manoeuvres are shown in Fig. 5. From the figures shown above, it can be inferred that the outer-loop MPC algorithm can generate the desired reference pair $\left(\boldsymbol{x}_{\text {ref }}, \boldsymbol{u}_{\text {ref }}\right)$ that satisfies the states and inputs constraints. With the help of 
the inner-loop stabilising controller, the quadrotor is able to asymptotically follow the constrained reference commands, such as the attitude angle commands, so as to achieve a favourable trajectory tracking performance.

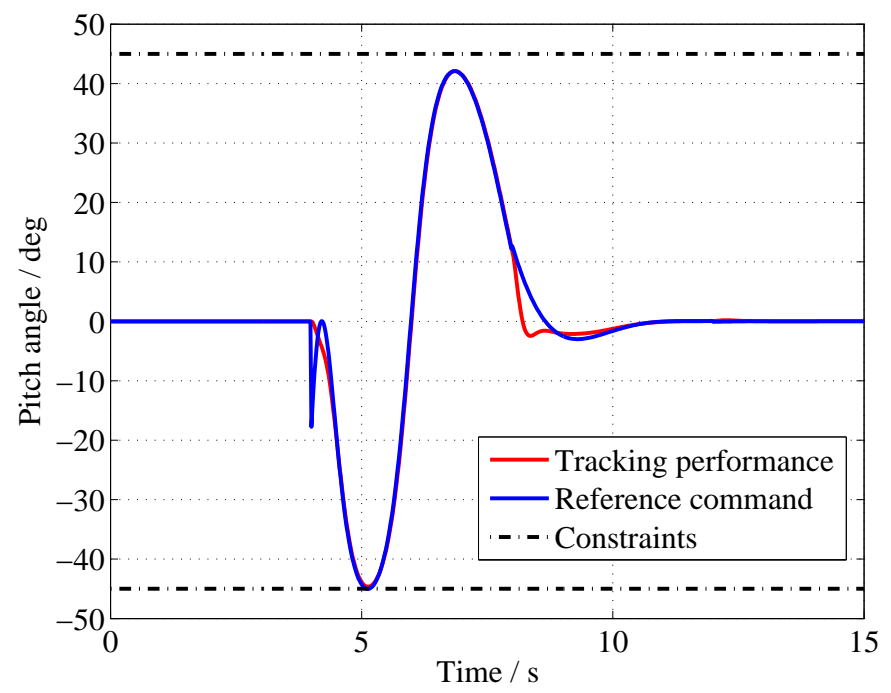

Figure 3. Pitch angle response to a $20 \mathrm{~m}$ step along $x$ direction

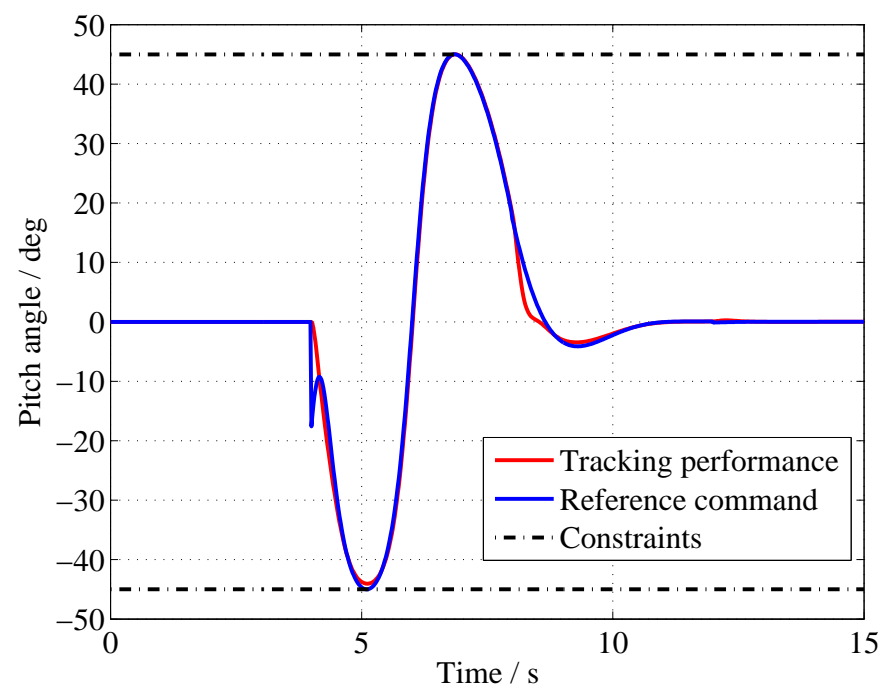

Figure 4. Pitch angle response to a $25 \mathrm{~m}$ step along $x$ direction

\section{2. $\quad$ Trajectory tracking under disturbances}

In this case study, the quadrotor is required to track a three-dimensional clockwise square trajectory, starting from and ending at the origin. For simplicity, the heading angle $\psi_{r}$ is controlled to remain constant. During the route, the lumped force and torque disturbances will be imposed on the quadrotor system to test the robustness of the proposed inner-loop anti- 


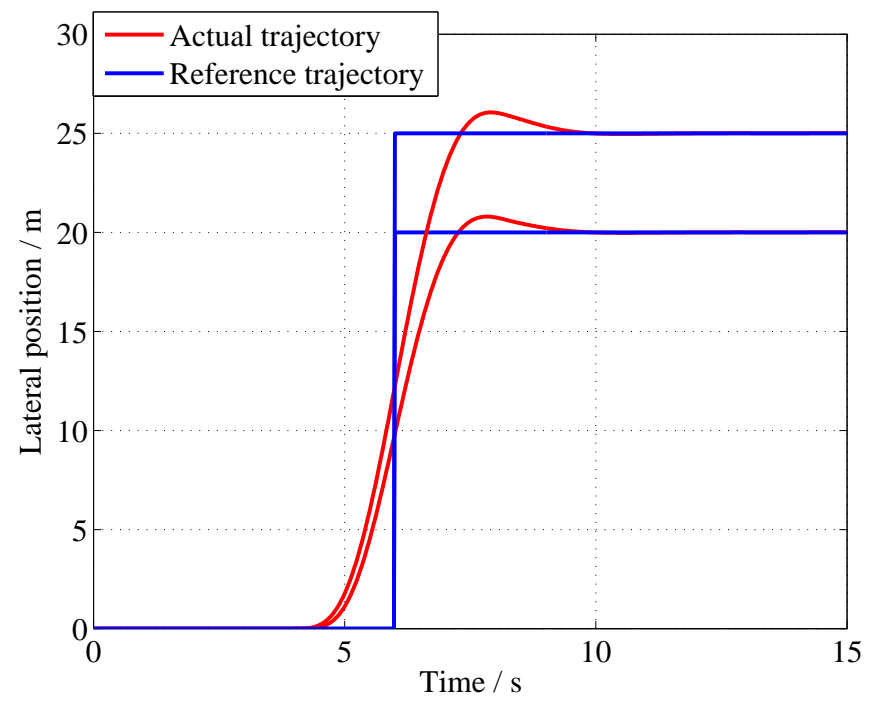

Figure 5. Position responses to the two steps

disturbance controller. As expressed in the Eq. (2), the force and torque disturbances are chosen as $\boldsymbol{F}_{\mathrm{d}}=\left[\begin{array}{llll}1 N & 1 N & 1 N\end{array}\right]^{\mathrm{T}}$ and $\boldsymbol{M}_{\mathrm{d}}=\left[\begin{array}{llll}0.1 N \cdot m & -0.1 N \cdot m & 0.1 N \cdot m\end{array}\right]^{\mathrm{T}}$, respectively. They are first applied at $1 \mathrm{~s}$ and then removed at $15 \mathrm{~s}$. At $20 \mathrm{~s}$, the disturbances are applied again and changed to $\boldsymbol{F}_{\mathrm{d}}=\left[\begin{array}{llll}-1 N & -1 N & -1 N\end{array}\right]^{\mathrm{T}}$ and $\boldsymbol{M}_{\mathrm{d}}=\left[\begin{array}{lll}-0.1 N \cdot m & 0.1 N \cdot m & -0.1 N \cdot m\end{array}\right]^{\mathrm{T}}$. To show the advantage of the CHADC method, the baseline $H_{\infty}$ controller with an integral action is also implemented in the inner-loop for comparative studies. The trajectory tracking performance of the box manoeuvre is shown in Fig. 6 in the 3D space. To be more specific, the horizontal projection of the tracking results are shown in Fig. 7 and the three position components $x-y-z$ are shown in Fig. 8. The corresponding control inputs of the two methods are given in Fig. 9 and Fig. 10. It can be seen that although the integral $H_{\infty}$ controller can stabilise the quadrotor to some extend, the control performance is affected by the disturbances. The suddenly appearing disturbances cause severe fluctuations in the actual trajectory. However, under the proposed constrained anti-disturbance scheme, the quadrotor can quickly response to the changes of disturbances at $1 s, 15 s$, and $20 s$ such that only small position variations are visible and followed by rapid recovery. Thus, this comparison demonstrates the promising robustness of the proposed constrained anti-disturbance scheme.

\section{Conclusion}

This paper proposes a constrained anti-disturbance control scheme which can be applied to differentially flat systems as demonstrated in quadrotor tracking control. The main motivation is driven by the requirement of constraining the system states and inputs to lie within an effective neighbourhood of the operating point in order to satisfy the linear control design. Intuitively, the MPC approach is considered to deal with the states and inputs constraints. The intrinsic properties of differential flatness and B-spline polynomials are exploited to transform the nonlinear optimisation into a QP problem, which significantly reduces the computational burden. The optimal states and inputs obtained by solving MPC online are used as the constrained reference for the inner-loop controller. To achieve the robustness against multiple disturbances, the CHADC method is employed to design an inner-loop controller, which includes DOBC and $H_{\infty}$ control to improve the disturbance rejection and attenuation abilities. Simulation results show that the quadrotor under the 


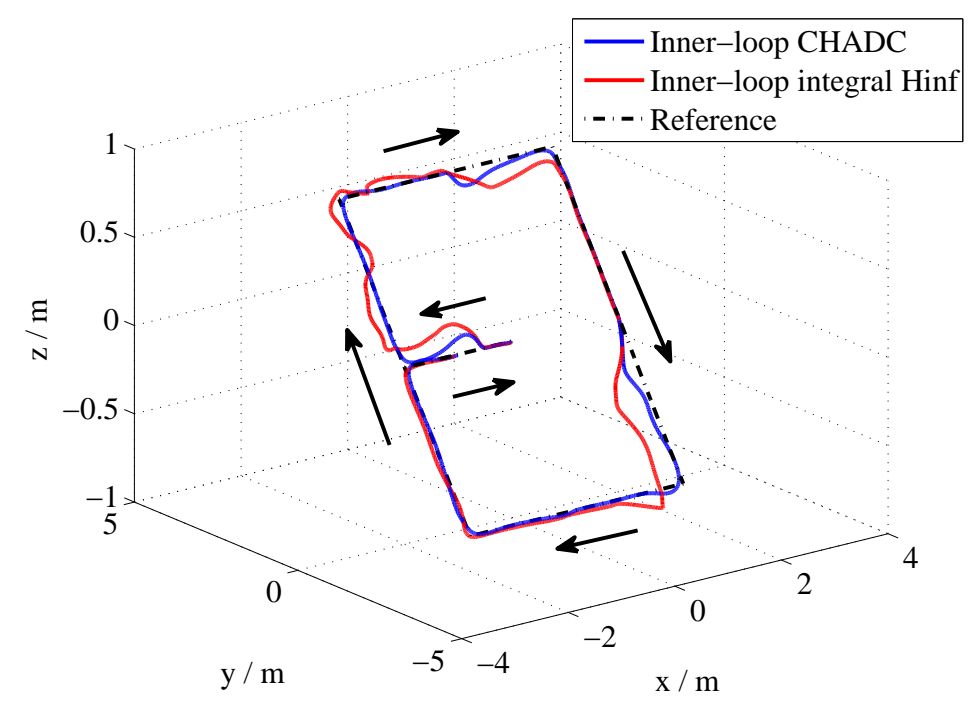

Figure 6. 3D trajectory tacking results

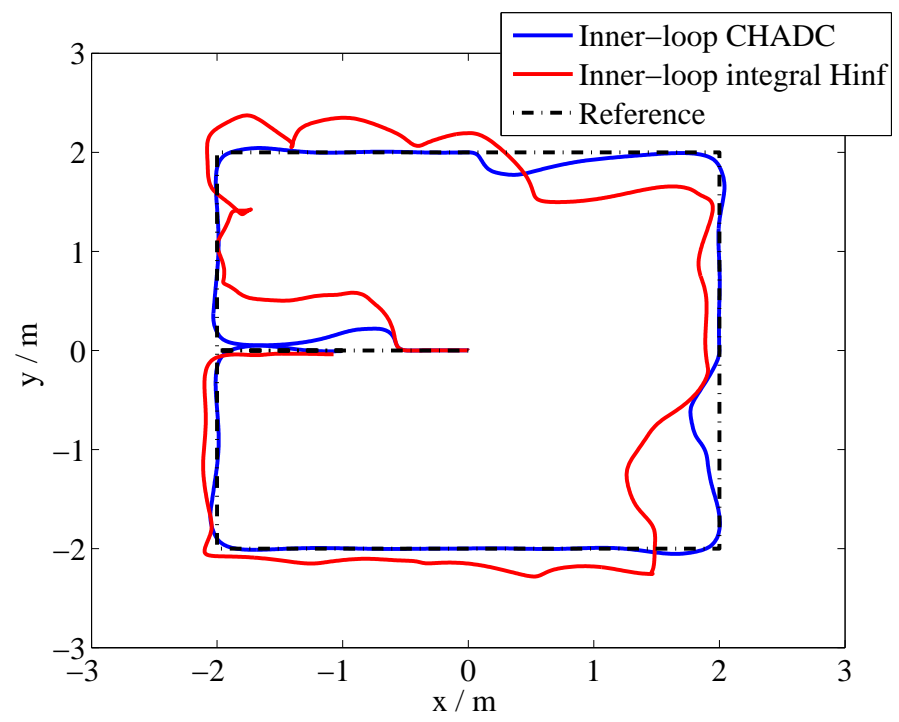

Figure 7. 2D trajectory tracking results

proposed control strategy satisfies the constraints during the aggressive manoeuvres and achieves good tracking performance against disturbances. This constrained anti-disturbance control scheme also can fit for other differential flat systems in the future.

\section{References}

Berry, A. J., Howitt, J., Gu, D.-W., \& Postlethwaite, I. (2011). A continuous local motion planning framework for unmanned vehicles in complex environments. Journal of Intelligent and Robotic Systems, 66 (4), 477494.

Chen, W.-H., Ballance, D. J., \& Gawthrop, P. J. (2003). Optimal control of nonlinear systems: a predictive control approach. Automatica, 39(4), 633-641. 

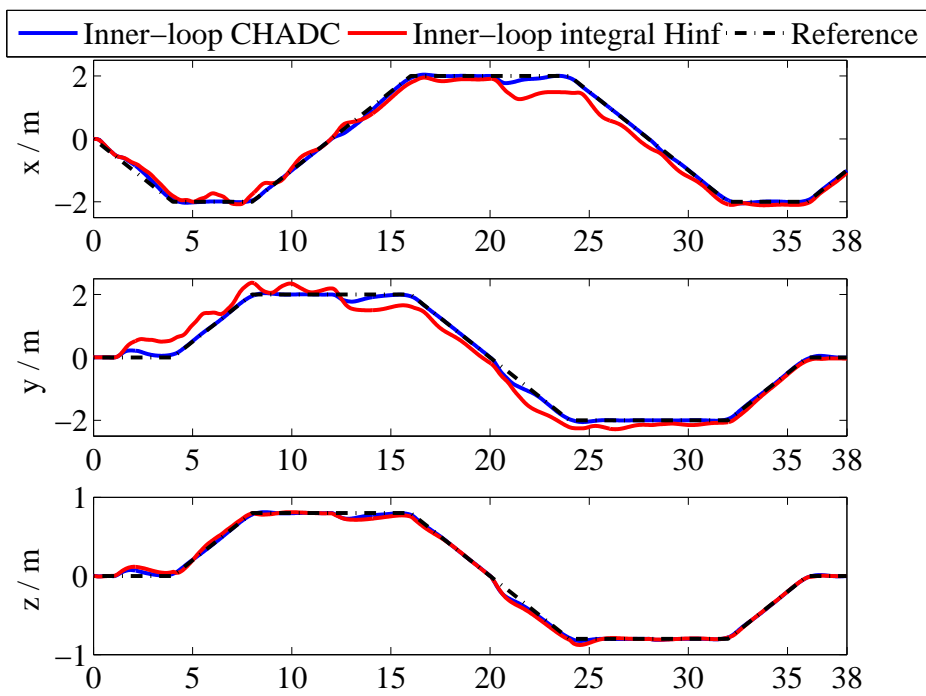

Figure 8. $\mathrm{x}-\mathrm{y}-\mathrm{z}$ components tracking results

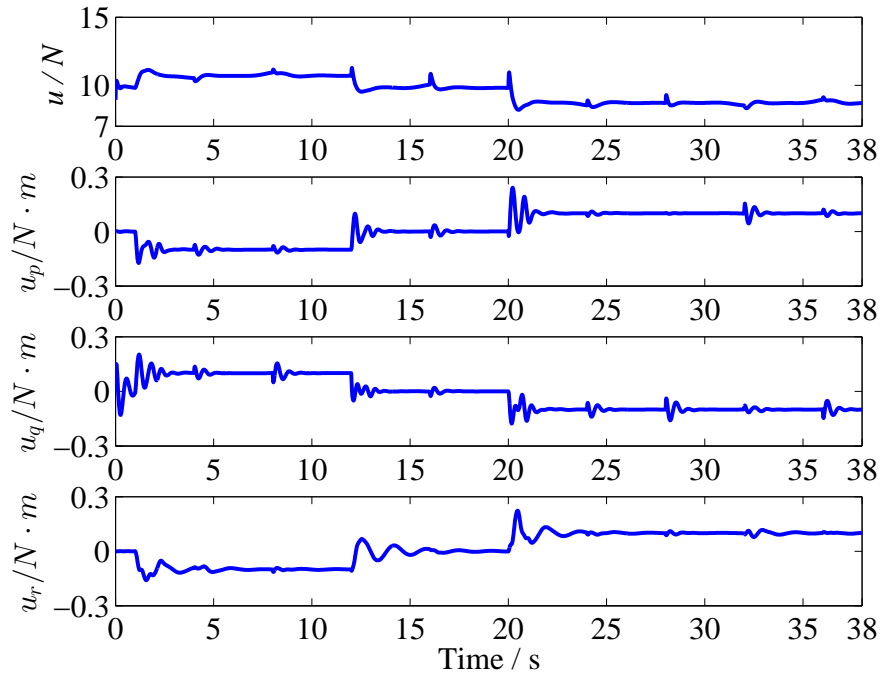

Figure 9. Control inputs under CHADC approach

Chen, W.-H., Ballance, D. J., Gawthrop, P. J., \& John, O. (2000). A nonlinear disturbance observer for robotic manipulators. IEEE Transcations on Industrial Electronics, 47(4), 932-938.

Chen, W.-H., Yang, J., Guo, L., \& Li, S. (2015). Disturbance observer-based control and related methods: an overview. IEEE Transactions on Industrial Electronics, 63(2), 1083-1095.

Cowling, I. D., Yakimenko, O. A., Whidborne, J. F., \& Cooke, A. K. (2010). Direct method based control system for an autonomous quadrotor. Journal of Intelligent and Robotic Systems, 60 (2), 285-316.

Faulwasser, T., Hagenmeyer, V., \& Findeisen, R. (2014). Constrained reachability and trajectory generation for flat systems. Automatica, 50(4), 1151-1159.

Fliess, M., Lvine, J., Martin, P., \& Rouchon, P. (1995). Flatness and defect of non-linear systems: introductory theory and examples. International Journal of Control, 61 (6), 1327-1361.

Flores, M. E., \& Milam, M. B. (2006). Trajectory generation for differentially flat systems via NURBS basis functions with obstacle avoidance. In Proceedings of the 2006 American Control Conference, Minneapolis, 


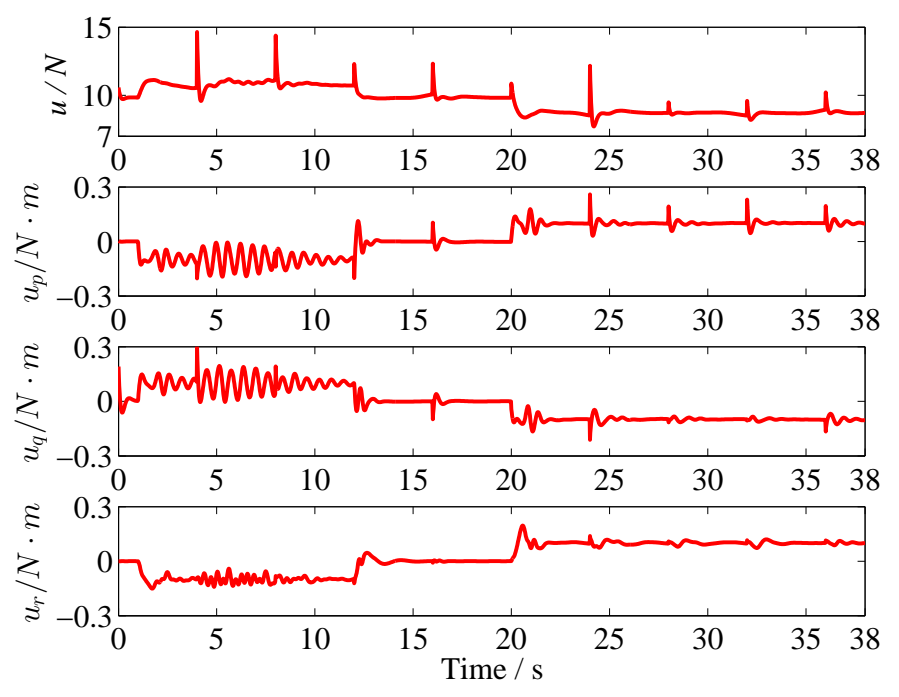

Figure 10. Control inputs under integral $H_{\infty}$ controller

$\mathrm{MN}, 5769-5775$.

Guo, L., \& Cao, S. (2013). Anti-disturbance control for systems with multiple disturbances. Boca Raton, FL: CRC Press.

Guo, L., \& Chen, W.-H. (2005). Disturbance attenuation and rejection for systems with nonlinearity via DOBC approach. International Journal of Robust and Nonlinear Control, 15(3), 109-125.

Guo, L., \& Wen, X.-Y. (2011). Hierarchical anti-disturbance adaptive control for non-linear systems with composite disturbances and applications to missile systems. Transactions of the Institute of Measurement and Control, 33(8), 942-956.

He, D.-F., Ji, H.-B., \& Yu, L. (2013). Constructive robust model predictive control for constrained non-linear systems with disturbances. IET Control Theory and Applications, 7(15), 1869-1876.

He, D.-F., Huang, H., \& Chen, Q.-X. (2014). Quasi-minmax MPC for constrained nonlinear systems with guaranteed input-to-state stability. Journal of the Franklin Institute, 351 (6), 3405-3423.

Hu, J., Wang, Z., Shen, B., \& Gao, H. (2013). Quantised recursive filtering for a class of nonlinear systems with multiplicative noises and missing measurements.International Journal of Control, 86 (4), 650-663.

Hu, J., Wang, Z., Liu, S., \& Gao, H. (2016). A variance-constrained approach to recursive state estimation for time-varying complex networks with missing measurements. Automatica, 64, 155-162.

Kendoul, F., Lara, D., Fantoni-Coichot, I., \& Lozano, R. (2007). Real-time nonlinear embedded control for an autonomous quadrotor helicopter. Journal of Guidance, Control, and Dynamics, 30 (4), 1049-1061.

Lu H., Liu C., Coombes M., Guo L., \& Chen W.-H. (2016). Online optimisation-based backstepping control design with application to quadrotor IET Control Theory and Applications, 10(14), 1601-1611.

Mahadevan, R., Agrawal, S. K., \& Doyle, F. J. (2001). Differential flatness based nonlinear predictive control of fed-batch bioreactors. Control Engineering Practice, 9(8), 889-899.

Mayne, D. Q., Rawlings, J. B., Rao, C. V., \& Scokaert, P. O. M. (2000). Constrained model predictive control: stability and optimality. Automatica, 36(6), 789-814.

Murray, R. M., Rathinam, M., \& Sluis, W. (1995). Differential flatness of mechanical control systems: a catalog of prototype systems. In Proceeding of ASME International Mechanical Engineering Congress and Exposition.

Peng, C., Fang, J., \& Xu, X. (2015). Mismatched disturbance rejection control for voltage-controlled active magnetic bearing via state-space disturbance observer. IEEE Transcations on Power Electronics, 30(5), $2753-2762$.

Piegl, L., \& Tiller, W. (1997). The NURBS book (2nd ed.). Berlin: Springer Verlag.

Prodan, I., Olaru, S., Bencatel, R., Borges de Sousa, J., Stoica, C., \& Niculescu, S.-I. (2013). Receding horizon flight control for trajectory tracking of autonomous aerial vehicles. Control Engineering Practice, 
$21(10), 1334-1349$.

Raptis, I. A., Valavanis, K. P., \& Vachtsevanos, G. J. (2012). Linear tracking control for small-scale unmanned helicopters. IEEE Transactions on Control Systems Technology, 20(4), 995-1010.

Suryawan, F., De Don, J., \& Seron, M. (2012). Splines and polynomial tools for flatness-based constrained motion planning. International Journal of Systems Science, 43(8), 1396-1411.

Wei, X., \& Guo, L. (2010). Composite disturbance-observer-based control and $H_{\infty}$ control for complex continuous models. International Journal of Robust and Nonlinear Control, 20(1), 106-118.

Wei, X., Zhang, H.-F., \& Guo, L. (2009). Composite disturbance-observer-based control and terminal sliding mode control for uncertain structural systems. International Journal of Systems Science, 40 (10), 10091017.

Yao, X., \& Guo, L. (2013). Composite anti-disturbance control for Markovian jump nonlinear systems via disturbance observer. Automatica, 49(8), 2538-2545.

Yang, J., Li, S., \& Yu, X. (2013). Sliding-mode control for systems with mismatched uncertainties via a disturbance observer. IEEE Transactions on Industrial Electronics, 60 (1), 160-169.

Yang, J., Zolotas, A., Chen, W.-H., Michail, K., \& Li, S. (2011). Robust control of nonlinear MAGLEV suspension system with mismatched uncertainties via DOBC approach. ISA Transactions, 50 (3), 389396. 